\title{
Well-posedness for a general class of differential inclusions
}

\author{
Sascha Trostorff*
}

\begin{abstract}
We consider an abstract class of differential inclusions, which covers differentialalgebraic and non-autonomous problems as well as problems with delay. Under weak assumptions on the operators involved, we prove the well-posedness of those differential inclusions in a pure Hilbert space setting. Moreover, we study the causality of the associated solution operator. The theory is illustrated by an application to a semistatic quasilinear variant of Maxwell's equations.
\end{abstract}

Keywords: differential inclusions, maximal monotone relations, non-autonomous problems, causality, Maxwell's equations

2010 MSC: $34 \mathrm{G} 25,46 \mathrm{~N} 20,35 \mathrm{~F} 60$

\section{Introduction}

In [18] it was shown that most (if not all) linear autonomous equations in mathematical physics share a common form, namely

$$
\left(\partial_{t} \mathcal{M}+\mathcal{A}\right) U=F
$$

where $\partial_{t}$ stands for the derivative with respect to time, $\mathcal{M}$ is a suitable bounded operator in space-time, which commutes with $\partial_{t}$ and $\mathcal{A}$ is a skew-selfadjoint operator in space, which in applications is a spatial differential operator. This result was generalised by the author in [26, 27, 25] to the case of differential inclusions, where the operator $\mathcal{A}$ is replaced by a maximal monotone relation. The resulting problem then takes the form

$$
(U, F) \in \partial_{t} \mathcal{M}+\mathcal{A} .
$$

This generalisation allows to study certain non-linear problems, especially equations describing physical phenomena with hysteresis effects. A particular case of an operator $\mathcal{M}$ is given by $\mathcal{M}=M_{0}+\partial_{t}^{-1} M_{1}$ for some bounded spatial operators $M_{0}, M_{1}$. The resulting problem then takes the simpler form

$$
(U, F) \in \partial_{t} M_{0}+M_{1}+\mathcal{A} .
$$

\footnotetext{
${ }^{*}$ Mathematisches Seminar, CAU Kiel, Germany, e-mail: trostorff@math.uni-kiel.de
} 
Replacing now $M_{0}, M_{1}$ by operator-valued multiplication operators, the resulting problem becomes non-autonomous. These problems were studied in [24] for the case of skewselfadjoint operators $\mathcal{A}$ and in [31] for the case of a maximal monotone relation $\mathcal{A}$. However, in the case of maximal monotone relations, the authors of [31] had to restrict the class of admissible multiplication operators $M_{0}$ and $M_{1}$. Finally, in [34 Waurick proved a well-posedness result for a very abstract class of non-autonomous differential equations, where $M_{0}$ and $M_{1}$ are replaced by arbitrary space-time operators $\mathcal{M}, \mathcal{N}$, where $\mathcal{M}$ should have a bounded commutator with $\partial_{t}$. With this result he was able to generalise both the results of [18] and [24]. However, it does not cover the result for the inclusions. It is the purpose of that article to provide a solution theory for differential inclusions of the form

$$
(U, F) \in \partial_{t} \mathcal{M}+\mathcal{N}+\mathcal{A},
$$

where $\mathcal{M}$ and $\mathcal{N}$ are space-time operators and $\mathcal{A}$ is a maximal monotone relation. We hereby generalise the results of [27, 31] and [34] and provide a unified solution theory for a broad class of problems.

Of course the problem of non-autonomous differential inclusions was studied in the literature before. We just mention some classical approaches, to tackle this problem. A standard approach for inclusions given in form of a Cauchy problem (i.e. $\mathcal{M}=1$ and time dependent $\mathcal{A}$ in (10) uses the concept of evolution families introduced by Kato [8] for evolution equations and generalised to inclusions by Crandall and Pazy in 3 . Other approaches use approximations by replacing the time derivative in (11) by the difference quotient and then proving that the corresponding solutions converge in a suitable sense (e.g. [5, 9]). A third approach uses the notion of integral solutions introduced by Bénilan in [1] for autonomous problems and generalised in [9] to the non-autonomous case.

In contrast to all these classical approaches, we assume the relation $\mathcal{A}$ to be timeindependent. The non-autonomous behaviour enters the equation via the bounded spacetime operators $\mathcal{M}$ and $\mathcal{N}$. This has the big advantage that we do not have any problems with time-dependent domains.

The article is structured as follows: In the next section we present the underlying Hilbert space framework and recall some basic facts of maximal monotone relations. Section 3 is devoted to the well-posedness result for inclusions of the form (11) in an exponentially weighted $L_{2}$-space. In Section 4 we prove under stronger assumptions the causality of the solution operator and its independence of the particular choice of the exponential weight (see Theorem 4.10 for the precise statement). Finally, we apply our results to a semistatic quasilinear variant of Maxwell's equations and thereby generalise the result of [12].

\section{Preliminaries}

We begin by introducing the Hilbert space setting we are working with throughout this article. Throughout let $H$ denote a Hilbert space (real or complex, in case of a real Hilbert space one can ignore all forthcoming occurring real parts) with inner product $\langle\cdot, \cdot\rangle$ and 
induced norm $|\cdot|$. Following [20, 18] we introduce the following weighted $L_{2}$-space and the derivative operator defined on it.

Definition. Let $\rho \geq 0$. We define the following space of (equivalence classes of) square integrable functions with respect to an exponentially weighted Lebesgue measure

$$
L_{2, \rho}(\mathbb{R} ; H):=\left\{f: \mathbb{R} \rightarrow H ; f \text { Bochner-measurable, } \int_{\mathbb{R}}|f(t)|^{2} \mathrm{e}^{-2 \rho t} \mathrm{~d} t<\infty\right\} .
$$

Clearly, $L_{2, \rho}(\mathbb{R} ; H)$ becomes a Hilbert space equipped with the usual inner product

$$
\langle f, g\rangle_{\rho}:=\int_{\mathbb{R}}\langle f(t), g(t)\rangle \mathrm{e}^{-2 \rho t} \mathrm{~d} t \quad\left(f, g \in L_{2, \rho}(\mathbb{R} ; H)\right) .
$$

We denote the induced norm by $|\cdot|_{\rho}$. Moreover, we define the operator $\partial_{t, \rho}$ as the closure of the operator

$$
C_{c}^{\infty}(\mathbb{R} ; H) \subseteq L_{2, \rho}(\mathbb{R} ; H) \rightarrow L_{2, \rho}(\mathbb{R} ; H): \phi \mapsto \phi^{\prime},
$$

where $C_{c}^{\infty}(\mathbb{R} ; H)$ denotes the space of $H$-valued arbitrarily differentiable functions on $\mathbb{R}$ with compact support.

Remark 2.1. (a) The domain of $\partial_{t, \rho}$ consists of those elements in $L_{2, \rho}(\mathbb{R} ; H)$ whose distributional derivative lies again in $L_{2, \rho}(\mathbb{R} ; H)$.

(b) For $\rho=0$ the space $L_{2,0}(\mathbb{R} ; H)$ is the usual $L_{2}$-space and the derivative $\partial_{t, 0}$ coincides with the classical weak derivative with domain $H^{1}(\mathbb{R} ; H)$.

We recall some basic properties of $\partial_{t, \rho}$ and refer to [7, 23] for the respective proofs.

Proposition 2.2. Let $\rho \geq 0$. Then the operator $\partial_{t, \rho}$ is normal with $\partial_{t, \rho}^{*}=-\partial_{t, \rho}+2 \rho$. Moreover, $\sigma\left(\partial_{t, \rho}\right)=\{\mathrm{i} t+\rho ; t \in \mathbb{R}\}$. In particular, if $\rho>0$, then $\partial_{t, \rho}$ is boundedly invertible with $\left\|\partial_{t, \rho}^{-1}\right\|=\frac{1}{\rho}$ and

$$
\left(\partial_{t, \rho}^{-1} f\right)(t)=\int_{-\infty}^{t} f(s) \mathrm{d} s \quad\left(f \in L_{2, \rho}(\mathbb{R} ; H), t \in \mathbb{R}\right) .
$$

Remark 2.3. It should be noted that the bounded invertibility of $\partial_{t, \rho}$ just holds, since we deal with the whole real line and not just with the positive real line or an interval. The main reason is that on the positive real line or an interval, we need to impose initial conditions to obtain invertibility of $\partial_{t, \rho}$. By dealing with the whole real line, we implicitly impose a vanishing initial condition at $-\infty$. For the treatment of differential inclusions on $\mathbb{R}_{\geq 0}$ within the framework introduced here, we refer to [26].

With this operator at hand, we are able to define a scale of associated Hilbert spaces called the Sobolev chain associated with $\partial_{t, \rho}$, [20] (see also [15, 4], where these spaces are called Sobolev towers). 
Proposition 2.4. Let $\rho>0$. For $k \in \mathbb{N}$ we define the spaces

$$
H_{\rho}^{k}(\mathbb{R} ; H):=\operatorname{dom}\left(\partial_{t, \rho}^{k}\right)
$$

equipped with the inner product

$$
\langle u, v\rangle_{\rho, k}:=\left\langle\partial_{t, \rho}^{k} u, \partial_{t, \rho}^{k} v\right\rangle_{\rho} \quad\left(u, v \in \operatorname{dom}\left(\partial_{t, \rho}^{k}\right)\right) .
$$

Moreover, we set $H_{\rho}^{-k}(\mathbb{R} ; H)$ as the completion of $L_{2, \rho}(\mathbb{R} ; H)$ with respect to the norm induced by

$$
\langle u, v\rangle_{\rho,-k}:=\left\langle\partial_{t, \rho}^{-k} u, \partial_{t, \rho}^{-k} v\right\rangle_{\rho} \quad\left(u, v \in L_{2, \rho}(\mathbb{R} ; H)\right) .
$$

For $k, j \in \mathbb{Z}$ with $k \geq j$ we have that

$$
H_{\rho}^{k}(\mathbb{R} ; H) \hookrightarrow H_{\rho}^{j}(\mathbb{R} ; H)
$$

and the operator

$$
\partial_{t, \rho}^{k-j}: C_{c}^{\infty}(\mathbb{R} ; H) \subseteq H_{\rho}^{k}(\mathbb{R} ; H) \rightarrow H_{\rho}^{j}(\mathbb{R} ; H)
$$

extends to a unitary operator again denoted by $\partial_{t, \rho}^{k-j}$.

We state a simple observation, which will be used several times later on.

Lemma 2.5. Let $\rho>0$ and $k, j \in \mathbb{Z}$ with $k \geq j$. Let $\left(u_{n}\right)_{n \in \mathbb{N}}$ be a sequence in $H_{\rho}^{k}(\mathbb{R} ; H)$ such that $u_{n} \rightarrow u$ in $H_{\rho}^{j}(\mathbb{R} ; H)$ for some $u \in H_{\rho}^{j}(\mathbb{R} ; H)$ and assume that $\left(u_{n}\right)_{n}$ is bounded in $H_{\rho}^{k}(\mathbb{R} ; H)$. Then $u \in H_{\rho}^{k}(\mathbb{R} ; H)$.

Proof. As $\left(u_{n}\right)_{n}$ is bounded in $H_{\rho}^{k}(\mathbb{R} ; H)$, we can assume without loss of generality that $u_{n} \rightarrow v$ for some $v \in H_{\rho}^{k}(\mathbb{R} ; H)$. As $H_{\rho}^{k}(\mathbb{R} ; H) \hookrightarrow H_{\rho}^{j}(\mathbb{R} ; H)$ we infer that $u_{n} \rightarrow v$ in $H_{\rho}^{j}(\mathbb{R} ; H)$ and hence, $u=v \in H_{\rho}^{k}(\mathbb{R} ; H)$.

Finally, we provide a useful characterisation for elements lying in $H_{\rho}^{1}(\mathbb{R} ; H)$.

Lemma 2.6 ([31, Proposition 2.1]). Let $\rho \geq 0, u \in L_{2, \rho}(\mathbb{R} ; H)$ and for $h>0$ we define

$$
D_{h} u:=\frac{1}{h}\left(\tau_{h} u-u\right) \in L_{2, \rho}(\mathbb{R} ; H),
$$

where $\tau_{h} u:=u(\cdot+h)$. Then, $u \in H_{\rho}^{1}(\mathbb{R} ; H)$ if and only if $\left(D_{h} u\right)_{h \in] 0,1]}$ is bounded in $L_{2, \rho}(\mathbb{R} ; H)$. In each case we have that

$$
D_{h} u \rightarrow \partial_{t, \rho} u \quad(h \rightarrow 0+)
$$

in $L_{2, \rho}(\mathbb{R} ; H)$.

Besides the presented Hilbert space setting, we need the framework of maximal monotone relations. For this topic and the proofs of the subsequent results we refer to the monographs [2, 14, 6]. 
Definition. A binary relation $A \subseteq H \times H$ is called monotone, if

$$
\forall(u, v),(x, y) \in A: \operatorname{Re}\langle u-x, v-y\rangle \geq 0 .
$$

$A$ is called maximal monotone if $A$ is monotone and for each monotone relation $B \subseteq$ $H \times H$ we have that

$$
A \subseteq B \Rightarrow A=B
$$

Moreover, we say that $A$ is $c$-(maximal) monotone for some $c \in \mathbb{R}$, if $A-c:=\{(u, v-$ $c u) ;(u, v) \in A\}$ is (maximal) monotone.

We need the following lifting result.

Lemma 2.7 ([2, Example 2.3.3]). Let $A \subseteq H \times H$ and $(\Omega, \Sigma, \mu)$ a measure space. We define

$$
A_{\mu}:=\left\{(u, v) \in L_{2}(\mu ; H) \times L_{2}(\mu ; H) ;(u(t), v(t)) \in A \quad(t \in \Omega \text { a.e. })\right\} .
$$

If $A$ is monotone, then so is $A_{\mu}$. Moreover, if $A$ is maximal monotone and $(0,0) \in A$ it follows that $A_{\mu}$ is maximal monotone as well.

Remark 2.8. If $\mu(\Omega)<\infty$ then the assumption $(0,0) \in A$ can be dropped. However, since we want to apply this lifting result to $\Omega=\mathbb{R}$ and $\mu=\mathrm{e}^{-2 \rho t} \mathrm{~d} t$, we need to impose this condition in our main result. If one just deals with $\mathbb{R}_{\geq 0}$ as time horizon, one obtains $\mu\left(\mathbb{R}_{\geq 0}\right)=\frac{1}{2 \rho}<\infty$ and hence, this additional condition is superfluous.

The maximal monotonicity of a monotone relation $A$ can be characterised by a range condition on $A$. This is the celebrated Theorem by Minty.

Theorem 2.9 (Minty, [13]). Let $A \subseteq H \times H$ be monotone. Then the following statements are equivalent:

(i) A is maximal monotone,

(ii) For each $\lambda>0$ and each $z \in H$ there exists $u \in H$ such that $(u, z) \in 1+\lambda A$, i.e. there is $v \in H$ with $(u, v) \in A$ and $u+\lambda v=z$.

(iii) There exists $\lambda>0$ such that for each $z \in H$ there exists $u \in H$ with $(u, z) \in 1+\lambda A$.

Example 2.10. For $\rho>0$ the operator $\partial_{t, \rho}$ is $\rho$-maximal monotone. The monotonicity follows by

$$
\left\langle\partial_{t, \rho} \phi, \phi\right\rangle_{\rho}=\left\langle\phi, \partial_{t, \rho}^{*} \phi\right\rangle_{\rho}=\left\langle\phi,-\partial_{t, \rho} \phi\right\rangle_{\rho}+2 \rho|\phi|_{\rho}^{2} \quad\left(\phi \in \operatorname{dom}\left(\partial_{t, \rho}\right)\right),
$$

which yields

$$
\operatorname{Re}\left\langle\partial_{t, \rho} \phi, \phi\right\rangle_{\rho} \geq \rho|\phi|_{\rho}^{2} \quad\left(\phi \in \operatorname{dom}\left(\partial_{t, \rho}\right)\right) .
$$

Moreover, for $f \in L_{2, \rho}(\mathbb{R} ; H)$ we set

$$
u:=\rho \partial_{t, \rho}^{-1} f \in \operatorname{dom}\left(\partial_{t, \rho}\right)
$$

and obtain

$$
u+\frac{1}{\rho}\left(\partial_{t, \rho}-\rho\right) u=\frac{1}{\rho} \partial_{t, \rho} u=f,
$$

which shows the maximal monotonicity by Theorem 2.9 . 
As a consequence of Minty's theorem and the definition of monotonicity we obtain the following proposition.

Proposition 2.11 ([2, Proposition 2.6]). Let $A \subseteq H \times H$ be maximal monotone. Then $(1+\lambda A)^{-1}$ is a Lipschitz-continuous mapping defined on the whole Hilbert space $H$ with 1 . $\left|(1+\lambda A)^{-1}\right|_{\text {Lip }} \leq 1$. Moreover, for each $\lambda>0$ the so-called Yosida-approximation

$$
A_{\lambda}:=\frac{1}{\lambda}\left(1-(1+\lambda A)^{-1}\right): H \rightarrow H
$$

is a monotone and Lipschitz-continuous mapping with $\left|A_{\lambda}\right|_{\text {Lip }} \leq \frac{1}{\lambda}$.

We conclude this section by some well-known perturbation results for maximal monotone relations.

Proposition 2.12. Let $A \subseteq H \times H$ be maximal monotone and $B: H \rightarrow H$ Lipschitz continuous. Moreover, assume that $A+B:=\{(x, y+B x) ;(x, y) \in A\}$ is monotone. Then $A+B$ is maximal monotone.

Proof. For $B=0$ there is nothing to show. So assume that $B \neq 0$. By Theorem 2.9 it suffices to prove that for $0<\lambda<\frac{1}{|B|_{\text {Lip }}}$ and each $z \in H$ there exists $u \in H$ with $(u, z) \in 1+\lambda(A+B)$. The latter is equivalent to

$$
u=(1+\lambda A)^{-1}(z-\lambda B u) .
$$

Since $(1+\lambda A)^{-1}$ is Lipschitz-continuous with $\left|(1+\lambda A)^{-1}\right|_{\text {Lip }} \leq 1$ and $|\lambda B|_{\text {Lip }}<1$ by the choice of $\lambda$, the assertion follows from the contraction mapping theorem.

Corollary 2.13. Let $B: H \rightarrow H$ be monotone and Lipschitz-continuous. Then $B$ is maximal monotone.

In particular, if $B \subseteq H \times H$ is maximal monotone, then its Yosida-approximation $B_{\lambda}$ is Lipschitz-continuous and monotone and hence, maximal monotone. Moreover, if $A \subseteq$ $H \times H$ is maximal monotone, then so is $A+B_{\lambda}$ for each $\lambda>0$ and hence, $\left(1+A+B_{\lambda}\right)^{-1}$ is a Lipschitz-continuous mapping. This observation can be used to prove the following perturbation result.

Proposition 2.14 ([6, Proposition 3.1]). Let $A, B \subseteq H \times H$ be two maximal monotone relations. Moreover, let $z \in H$. Then there exists $u \in H$ such that $(u, z) \in 1+A+B$ (i.e., there are $v, w \in H$ with $(u, v) \in A,(u, w) \in B$ and $u+v+w=z)$ if and only if

$$
\sup _{\lambda>0}\left|B_{\lambda}\left(u_{\lambda}\right)\right|<\infty
$$

where $u_{\lambda}:=\left(1+A+B_{\lambda}\right)^{-1}(z)$. In the latter case, $u_{\lambda} \rightarrow u$ as $\lambda \rightarrow 0+$.

With the help of the latter proposition, one can prove the following perturbation result.

\footnotetext{
${ }^{1}$ We denote the smallest Lipschitz-constant for a Lipschitz continuous mapping $f$ by $|f|_{\text {Lip }}$.
} 
Corollary 2.15 ([25, Proposition 1.22]). Let $A, B \subseteq H \times H$ be two maximal monotone relations. Moreover, assume that $A$ is bounded, i.e. for each $U \subseteq H$ bounded, the set

$$
\{v \in H ; \exists u \in U:(u, v) \in A\}
$$

is bounded. If $\operatorname{dom}(A) \cap \operatorname{dom}(B) \neq \emptyset$ (i.e. $\exists u, v, w \in H:(u, v) \in A$ and $(u, w) \in B$ ) then $A+B$ is maximal monotone.

\section{The main result}

Throughout, let $\rho>0$. We begin with stating the hypotheses of the operators and relations involved, which we assume to be valid throughout this section.

Hypotheses A. Let $\mathcal{M}, \mathcal{N}, \mathcal{M}^{\prime} \in L\left(L_{2, \rho}(\mathbb{R} ; H)\right)$ be such that:

(a) $\mathcal{M} \partial_{t, \rho} \subseteq \partial_{t, \rho} \mathcal{M}-\mathcal{M}^{\prime}$

(b) There exists $c>0$ such that

$$
\operatorname{Re}\left\langle\left(\partial_{t, \rho} \mathcal{M}+\mathcal{N}\right) \varphi, \varphi\right\rangle_{\rho} \geq c|\varphi|_{\rho}^{2}
$$

for each $\varphi \in C_{c}^{\infty}(\mathbb{R} ; H)$.

Moreover, let $\mathcal{A} \subseteq L_{2, \rho}(\mathbb{R} ; H) \times L_{2, \rho}(\mathbb{R} ; H)$ be maximal monotone such that

$$
\forall u, v \in L_{2, \rho}(\mathbb{R} ; H):\left((u, v) \in \mathcal{A} \Rightarrow \forall h \geq 0:\left(\tau_{h} u, \tau_{h} v\right) \in \mathcal{A}\right) .
$$

It is noteworthy that the perspective on differential inclusions presented here, is that the relation $\mathcal{A}$ is independent of time in the sense that it commutes with time translation, while the time-dependence enter the problem via coefficients, which are incorporated in the operators $\mathcal{M}$ and $\mathcal{N}$. This is the standard case in problems arising in mathematical physics.

Our main theorem reads as follows.

Theorem 3.1 (Well-posedness). The relation $\overline{\partial_{t, \rho} \mathcal{M}+\mathcal{N}+\mathcal{A}}$ is c-maximal monotone. In particular, the inverse relation

$$
\mathcal{S}_{\rho}:=\left(\overline{\partial_{t, \rho} \mathcal{M}+\mathcal{N}+\mathcal{A}}\right)^{-1}: L_{2, \rho}(\mathbb{R} ; H) \rightarrow L_{2, \rho}(\mathbb{R} ; H)
$$

is a Lipschitz-continuous mapping with $\left|\mathcal{S}_{\rho}\right|_{\text {Lip }} \leq \frac{1}{c}$. Hence, for each $f \in L_{2, \rho}(\mathbb{R} ; H)$ there exists a unique $u \in L_{2, \rho}(\mathbb{R} ; H)$ with

$$
(u, f) \in \overline{\partial_{t, \rho} \mathcal{M}+\mathcal{N}+\mathcal{A}}
$$

and $u$ depends Lipschitz-continuously on $f$.

Before we come to the proof of this theorem, we illustrate the hypotheses by some concrete examples for the operators $\mathcal{M}$ and $\mathcal{N}$. 
Example 3.2. (a) If $\mathcal{N}=0$ and $\mathcal{M}$ is given in terms of an operator-valued analytic function of $\partial_{t, \rho}$, then condition (a) is trivially satisfied with $\mathcal{M}^{\prime}=0$ and the resulting problem becomes autonomous. This class of operators was introduced in [18] and allows the treatment of a broad class of differential equations arising in mathematical physics (see e.g. [18, 10, 19, 22]) and includes different types of equations, such as fractional differential equations (see [21]), delay equations with finite and infinite delay (see [7]), and integro-differential equations (see [29]).

(b) A second class of problems covered by the hypotheses is given by non-autonomous equations, which are local with respect to time. More precisely, $\mathcal{M}, \mathcal{N}$ are given as operator-valued multiplication operators, such that $\mathcal{M}$ is Lipschitz-continuous. Then, Rademacher's theorem implies that (a) holds. Such equations were studied in [24] and generalised to inclusions in [31].

(c) The hypotheses also allow the treatment of non-autonomous differential inclusions which are non-local in space and time. A classical example would be integral operators of the form

$$
(\mathcal{M} u)(t):=\int_{\mathbb{R}} k(t, s) u(s) \mathrm{d} s \quad(t \in \mathbb{R})
$$

with a suitable (possibly operator-valued) kernel $k$. Indeed, if $k$ is differentiable with respect to the first variable and satisfies suitable integrability conditions, one easily can show that (a) holds. To find the right conditions on $k$ to ensure (b) is more delicate and will be postponed to future research. In case of a kernel $k(t, s)=k(t-s)$, this was done in [29, 30] even for operator-valued kernels.

We begin by proving Theorem 3.1 in the case $\mathcal{A}=0$. The proof follows the rationale of [35, Section 3.3]. For the readers convenience we recall the definition of a core of a closed operator.

Definition. Let $S: \operatorname{dom}(S) \subseteq X \rightarrow Y$ be a closed linear operator between two normed spaces $X$ and $Y$. A linear subspace $D \subseteq X$ is called a core for $S$, if $S=\overline{\left.S\right|_{D}}$.

Proposition 3.3. $C_{c}^{\infty}(\mathbb{R} ; H)$ is a core for $\partial_{t, \rho} \mathcal{M}$. Moreover, the operator $\partial_{t, \rho} \mathcal{M}+\mathcal{N}$ is c-maximal monotone.

Proof. First, we observe that $\left(1+\varepsilon \partial_{t, \rho}\right)^{-1} \rightarrow 1$ strongly as $\varepsilon \rightarrow 0+$. Indeed, since $\partial_{t, \rho}$ is maximal monotone by Example 2.10 we have $\left\|\left(1+\varepsilon \partial_{t, \rho}\right)^{-1}\right\| \leq 1$ for each $\varepsilon>0$. Thus, it suffices to prove the strong convergence on a dense subset of $L_{2, \rho}(\mathbb{R} ; H)$. Since for $u \in \operatorname{dom}\left(\partial_{t, \rho}\right)$ we have that

$$
\left(1+\varepsilon \partial_{t, \rho}\right)^{-1} u-u=-\varepsilon\left(1+\varepsilon \partial_{t, \rho}\right)^{-1} \partial_{t, \rho} u \rightarrow 0 \quad(\varepsilon \rightarrow 0+)
$$

the assertion follows.

We now prove that $\operatorname{dom}\left(\partial_{t, \rho}\right)$ is a core for $\partial_{t, \rho} \mathcal{M}$. More precisely, we show that for $u \in \operatorname{dom}\left(\partial_{t, \rho} \mathcal{M}\right)$ and $u_{\varepsilon}:=\left(1+\varepsilon \partial_{t, \rho}\right)^{-1} u$ with $\varepsilon>0$ we have that

$$
\partial_{t, \rho} \mathcal{M} u_{\varepsilon} \rightarrow \partial_{t, \rho} \mathcal{M} u \quad(\varepsilon \rightarrow 0+) .
$$


Indeed, we have that

$$
\begin{aligned}
\partial_{t, \rho} \mathcal{M} u_{\varepsilon} & =\partial_{t, \rho} \mathcal{M}\left(1+\varepsilon \partial_{t, \rho}\right)^{-1} u \\
& =\left(1+\varepsilon \partial_{t, \rho}\right)^{-1} \partial_{t, \rho} \mathcal{M} u+\varepsilon \partial_{t, \rho}\left(1+\varepsilon \partial_{t, \rho}\right)^{-1} \mathcal{M}^{\prime}\left(1+\varepsilon \partial_{t, \rho}\right)^{-1} u \\
& =\left(1+\varepsilon \partial_{t, \rho}\right)^{-1} \partial_{t, \rho} \mathcal{M} u+\mathcal{M}^{\prime}\left(1+\varepsilon \partial_{t, \rho}\right)^{-1} u-\left(1+\varepsilon \partial_{t, \rho}\right)^{-1} \mathcal{M}^{\prime}\left(1+\varepsilon \partial_{t, \rho}\right)^{-1} u \\
& \rightarrow \partial_{t, \rho} \mathcal{M} u \quad(\varepsilon \rightarrow 0+) .
\end{aligned}
$$

Hence, it suffices to approximate elements $u \in \operatorname{dom}\left(\partial_{t, \rho}\right)$ by a sequence $\left(\varphi_{n}\right)_{n}$ in $C_{c}^{\infty}(\mathbb{R} ; H)$ such that $\varphi_{n} \rightarrow u$ and $\partial_{t, \rho} \mathcal{M} \varphi_{n} \rightarrow \partial_{t, \rho} \mathcal{M} u$ in $L_{2, \rho}(\mathbb{R} ; H)$. For doing so, we choose a sequence $\left(\varphi_{n}\right)_{n}$ in $C_{c}^{\infty}(\mathbb{R} ; H)$ such that $\varphi_{n} \rightarrow u$ in $H_{\rho}^{1}(\mathbb{R} ; H)$. Then, in particular, $\varphi_{n} \rightarrow u$ in $L_{2, \rho}(\mathbb{R} ; H)$ and

$$
\partial_{t, \rho} \mathcal{M} \varphi_{n}=\mathcal{M} \partial_{t, \rho} \varphi_{n}+\mathcal{M}^{\prime} \varphi_{n} \rightarrow \mathcal{M} \partial_{t, \rho} u+\mathcal{M}^{\prime} u=\partial_{t, \rho} \mathcal{M} u
$$

We now prove the maximal monotonicity of $\partial_{t, \rho} \mathcal{M}+\mathcal{N}-c$. As $C_{c}^{\infty}(\mathbb{R} ; H)$ is a core for this operator, the monotonicity follows by Hypotheses $\mathrm{A}(\mathrm{b})$. We claim that $C_{c}^{\infty}(\mathbb{R} ; H)$ is also a core for $\left(\partial_{t, \rho} \mathcal{M}+\mathcal{N}\right)^{*}$. Assuming that this is true, it follows from Hypotheses A (b) that $\left(\partial_{t, \rho} \mathcal{M}+\mathcal{N}\right)^{*}$ is one-to-one and hence, $\partial_{t, \rho} \mathcal{M}+\mathcal{N}$ has dense range. Since $\partial_{t, \rho} \mathcal{M}+\mathcal{N}$ is also closed and its inverse is bounded by what we have shown before, $\partial_{t, \rho} \mathcal{M}+\mathcal{N}$ is indeed onto and thus, $\partial_{t, \rho} \mathcal{M}+\mathcal{N}-c$ is maximal monotone. Thus, we are left to show that $C_{c}^{\infty}(\mathbb{R} ; H)$ is a core for $\left(\partial_{t, \rho} \mathcal{M}+\mathcal{N}\right)^{*}$. For showing this, we observe that

$$
\left(\partial_{t, \rho} \mathcal{M}+\mathcal{N}\right)^{*}=\left(\partial_{t, \rho} \mathcal{M}\right)^{*}+\mathcal{N}^{*}=\left(\mathcal{M} \partial_{t, \rho}+\mathcal{M}^{\prime}\right)^{*}+\mathcal{N}^{*}=\partial_{t, \rho}^{*} \mathcal{M}^{*}+\left(\mathcal{M}^{\prime}\right)^{*}+\mathcal{N}^{*}
$$

where we have used that $C_{c}^{\infty}(\mathbb{R} ; H)$ is a core for $\partial_{t, \rho} \mathcal{M}$. Thus, it suffices to show that $C_{c}^{\infty}(\mathbb{R} ; H)$ is a core for $\partial_{t, \rho}^{*} \mathcal{M}^{*}$. However, since

$$
\mathcal{M}^{*} \partial_{t, \rho}^{*} \subseteq\left(\partial_{t, \rho} \mathcal{M}\right)^{*}=\partial_{t, \rho}^{*} \mathcal{M}^{*}+\left(\mathcal{M}^{\prime}\right)^{*}
$$

we can follow the same lines as above and obtain the assertion.

In order to prove Theorem 3.1 we adopt the idea presented in [31] and first prove the well-posedness of an auxiliary problem. The well-posedness of the original problem will then follow by the perturbation result Proposition 2.12, The auxiliary problem reads as follows

$$
(u, f) \in \overline{\partial_{t, \rho} \mathcal{M}-\mathcal{M}^{\prime}+\delta+\mathcal{A}}
$$

for a suitable $\delta>0$.

Lemma 3.4. Let $\mathcal{L} \in L\left(L_{2, \rho}(\mathbb{R} ; H)\right)$ and $\delta>0$. Then $\partial_{t, \rho} \mathcal{M}+\mathcal{L}+\delta$ is $(c+\delta-\|\mathcal{L}-\mathcal{N}\|)$ maximal monotone.

Proof. For $u \in \operatorname{dom}\left(\partial_{t, \rho} \mathcal{M}\right)$ we have that

$$
\begin{aligned}
\operatorname{Re}\left\langle\left(\partial_{t, \rho} \mathcal{M}+\mathcal{L}+\delta\right) u, u\right\rangle_{\rho} & =\operatorname{Re}\left\langle\left(\partial_{t, \rho} \mathcal{M}+\mathcal{N}\right) u, u\right\rangle_{\rho}+\langle(\delta+(\mathcal{L}-\mathcal{N})) u, u\rangle_{\rho} \\
& \geq(c+\delta-\|\mathcal{L}-\mathcal{N}\|)|u|_{\rho}^{2},
\end{aligned}
$$


where we have used Proposition 3.3. Moreover, since

$$
\partial_{t, \rho} \mathcal{M}+\mathcal{L}+\delta-(c+\delta-\|\mathcal{L}-\mathcal{N}\|)=\left(\partial_{t, \rho} \mathcal{M}+\mathcal{N}-c\right)+(\|\mathcal{L}-\mathcal{N}\|+\mathcal{L}-\mathcal{N})
$$

the assertion follows by Proposition 2.12.

As an immediate consequence we derive the following proposition.

Proposition 3.5. Let $\delta>\left\|\mathcal{M}^{\prime}+\mathcal{N}\right\|$. Then $\partial_{t, \rho} \mathcal{M}-\mathcal{M}^{\prime}+\delta+\mathcal{A}$ is c-monotone and hence, $\left(\partial_{t, \rho} \mathcal{M}-\mathcal{M}^{\prime}+\delta+\mathcal{A}\right)^{-1}$ is a Lipschitz-continuous mapping defined on some subset of $L_{2, \rho}(\mathbb{R} ; H)$.

Thus, in order to prove that (2) is well-posed, it suffices to prove that the domain of $\left(\partial_{t, \rho} \mathcal{M}-\mathcal{M}^{\prime}+\delta+\mathcal{A}\right)^{-1}$ is dense in $L_{2, \rho}(\mathbb{R} ; H)$. We will prove that for each $f \in$ $H_{\rho}^{1}(\mathbb{R} ; H)$ there exists $u \in H_{\rho}^{1}(\mathbb{R} ; H)$ such that

$$
(u, f) \in \partial_{t, \rho} \mathcal{M}-\mathcal{M}^{\prime}+\delta+\mathcal{A},
$$

which in particular would give

$$
H_{\rho}^{1}(\mathbb{R} ; H) \subseteq \operatorname{dom}\left(\left(\partial_{t, \rho} \mathcal{M}-\mathcal{M}^{\prime}+\delta+\mathcal{A}\right)^{-1}\right)
$$

and thus, the well-posedness of (2) would follow. For doing so, let $f \in H_{\rho}^{1}(\mathbb{R} ; H)$ and define

$$
u_{\lambda}:=\left(\partial_{t, \rho} \mathcal{M}-\mathcal{M}^{\prime}+\delta+\mathcal{A}_{\lambda}\right)^{-1}(f) \quad(\lambda>0) .
$$

Note that $\left(\partial_{t, \rho} \mathcal{M}-\mathcal{M}^{\prime}+\delta+\mathcal{A}_{\lambda}\right)^{-1}$ with $\delta>\left\|\mathcal{M}^{\prime}+\mathcal{N}\right\|$ is a Lipschitz-continuous mapping on $L_{2, \rho}(\mathbb{R} ; H)$ defined on the whole space by Proposition 2.12 and Lemma 3.4 and thus $u_{\lambda} \in L_{2, \rho}(\mathbb{R} ; H)$ is defined. Our first goal is to prove the following theorem.

Theorem 3.6. Let $\delta>\left\|\mathcal{M}^{\prime}\right\|+\|\mathcal{N}\|$ and $f \in H_{\rho}^{1}(\mathbb{R} ; H)$. For $\lambda>0$ set

$$
u_{\lambda}:=\left(\partial_{t, \rho} \mathcal{M}-\mathcal{M}^{\prime}+\delta+\mathcal{A}_{\lambda}\right)^{-1}(f) .
$$

Then $u_{\lambda} \in H_{\rho}^{1}(\mathbb{R} ; H)$ and

$$
\left|u_{\lambda}\right|_{\rho, 1} \leq \frac{1}{c}|f|_{\rho, 1} .
$$

In order to prove this theorem, we define the following mapping

$$
\mathcal{B}_{\lambda}:=\partial_{t, \rho} \mathcal{A}_{\lambda} \partial_{t, \rho}^{-1}: H_{\rho}^{-1}(\mathbb{R} ; H) \rightarrow H_{\rho}^{-1}(\mathbb{R} ; H) .
$$

Then, by unitary equivalence, $\mathcal{B}_{\lambda}$ is maximal monotone and Lipschitz-continuous on $H_{\rho}^{-1}(\mathbb{R} ; H)$ with $\left|\mathcal{B}_{\lambda}\right|_{\text {Lip }} \leq \frac{1}{\lambda}$. However, we can also interpret $\mathcal{B}_{\lambda}$ as a mapping on $L_{2, \rho}(\mathbb{R} ; H)$ as the following proposition shows. 
Proposition 3.7. Let $\lambda>0$ and define

$$
\mathcal{B}_{\lambda}:=\partial_{t, \rho} \mathcal{A}_{\lambda} \partial_{t, \rho}^{-1}: H_{\rho}^{-1}(\mathbb{R} ; H) \rightarrow H_{\rho}^{-1}(\mathbb{R} ; H) .
$$

Then $\mathcal{B}_{\lambda}\left[L_{2, \rho}(\mathbb{R} ; H)\right] \subseteq L_{2, \rho}(\mathbb{R} ; H)$ and for $u \in L_{2, \rho}(\mathbb{R} ; H)$ we have that

$$
\begin{aligned}
\operatorname{Re}\left\langle\mathcal{B}_{\lambda}(u), u\right\rangle_{\rho} & \geq 0 \\
\left|\mathcal{B}_{\lambda}(u)\right|_{\rho} & \leq \frac{1}{\lambda}|u|_{\rho} .
\end{aligned}
$$

Proof. Let $u \in L_{2, \rho}(\mathbb{R} ; H)$. In order to prove $\mathcal{B}_{\lambda}(u) \in L_{2, \rho}(\mathbb{R} ; H)$ we need to show that $\mathcal{A}_{\lambda}\left(\partial_{t, \rho}^{-1} u\right) \in H_{\rho}^{1}(\mathbb{R} ; H)$. For doing so, we use the notation from Lemma 2.6 and estimate for $h>0$

$$
\begin{aligned}
\left|D_{h} \mathcal{A}_{\lambda}\left(\partial_{t, \rho}^{-1} u\right)\right|_{\rho} & =\frac{1}{h}\left|\tau_{h} \mathcal{A}_{\lambda}\left(\partial_{t, \rho}^{-1} u\right)-\mathcal{A}_{\lambda}\left(\partial_{t, \rho}^{-1} u\right)\right|_{\rho} \\
& =\frac{1}{h}\left|\mathcal{A}_{\lambda}\left(\tau_{h} \partial_{t, \rho}^{-1} u\right)-\mathcal{A}_{\lambda}\left(\partial_{t, \rho}^{-1} u\right)\right|_{\rho} \\
& \leq \frac{1}{\lambda}\left|D_{h} \partial_{t, \rho}^{-1} u\right|_{\rho} .
\end{aligned}
$$

As $\partial_{t, \rho}^{-1} u \in H_{\rho}^{1}(\mathbb{R} ; H)$ the latter estimate shows $\mathcal{A}_{\lambda}\left(\partial_{t, \rho}^{-1} u\right) \in H_{\rho}^{1}(\mathbb{R} ; H)$ according to Lemma 2.6. Furthermore, letting $h$ tend to 0 , we also infer that

$$
\left|\mathcal{B}_{\lambda}(u)\right|_{\rho}=\lim _{h \rightarrow 0+}\left|D_{h} \mathcal{A}_{\lambda}\left(\partial_{t, \rho}^{-1} u\right)\right|_{\rho} \leq \frac{1}{\lambda} \lim _{h \rightarrow 0+}\left|D_{h} \partial_{t, \rho}^{-1} u\right|_{\rho}=\frac{1}{\lambda}|u|_{\rho}
$$

Moreover, again by using Lemma 2.6, we estimate

$$
\begin{aligned}
\operatorname{Re}\left\langle\mathcal{B}_{\lambda}(u), u\right\rangle_{\rho} & =\operatorname{Re}\left\langle\partial_{t, \rho} \mathcal{A}_{\lambda}\left(\partial_{t, \rho}^{-1} u\right), \partial_{t, \rho} \partial_{t, \rho}^{-1} u\right\rangle_{\rho} \\
& =\lim _{h \rightarrow 0+} \frac{1}{h^{2}} \operatorname{Re}\left\langle\tau_{h} \mathcal{A}_{\lambda}\left(\partial_{t, \rho}^{-1} u\right)-\mathcal{A}_{\lambda}\left(\partial_{t, \rho}^{-1} u\right), \tau_{h} \partial_{t, \rho}^{-1} u-\partial_{t, \rho}^{-1} u\right\rangle_{\rho} \\
& =\lim _{h \rightarrow 0+} \frac{1}{h^{2}} \operatorname{Re}\left\langle\mathcal{A}_{\lambda}\left(\tau_{h} \partial_{t, \rho}^{-1} u\right)-\mathcal{A}_{\lambda}\left(\partial_{t, \rho}^{-1} u\right), \tau_{h} \partial_{t, \rho}^{-1} u-\partial_{t, \rho}^{-1} u\right\rangle_{\rho} \\
& \geq 0,
\end{aligned}
$$

where we have used the monotonicity of $\mathcal{A}_{\lambda}$.

The reason for considering the mapping $\mathcal{B}_{\lambda}$ is the following. Assume that $u_{\lambda}$ is given by (3). Then, at least formally, $\partial_{t, \rho} u$ satisfies

$$
\left(\partial_{t, \rho} \mathcal{M}+\delta+\mathcal{B}_{\lambda}\right)\left(\partial_{t, \rho} u\right)=\partial_{t, \rho} f
$$

Thus, we are led to consider the differential equation

$$
\left(\partial_{t, \rho} \mathcal{M}+\delta+\mathcal{B}_{\lambda}\right)(v)=g .
$$

Since $\mathcal{B}_{\lambda}$ is Lipschitz-continuous and maximal monotone on $H_{\rho}^{-1}(\mathbb{R} ; H)$, the latter equation is well-posed in $H_{\rho}^{-1}(\mathbb{R} ; H)$, if we can show that $\partial_{t, \rho} \mathcal{M}+\delta$ is $c$-maximal monotone on $H_{\rho}^{-1}(\mathbb{R} ; H)$ in some sense. This will be shown in the next proposition. 
Proposition 3.8. Let $\delta>\left\|\mathcal{M}^{\prime}\right\|+\|\mathcal{N}\|$. Then the mapping

$$
\partial_{t, \rho} \mathcal{M}+\delta: L_{2, \rho}(\mathbb{R} ; H) \subseteq H_{\rho}^{-1}(\mathbb{R} ; H) \rightarrow H_{\rho}^{-1}(\mathbb{R} ; H)
$$

is closable and its closure, denoted by $\mathcal{T}_{\delta}$, is $\left(c+\delta-\left\|\mathcal{M}^{\prime}+\mathcal{N}\right\|\right)$-maximal monotone.

Proof. To prove the closability, let $\left(v_{n}\right)_{n \in \mathbb{N}}$ be a sequence in $L_{2, \rho}(\mathbb{R} ; H)$ such that $v_{n} \rightarrow 0$ and $\left(\partial_{t, \rho} \mathcal{M}+\delta\right) v_{n} \rightarrow y$ for some $y \in H_{\rho}^{-1}(\mathbb{R} ; H)$, where both convergences are with respect to the topology in $H_{\rho}^{-1}(\mathbb{R} ; H)$. Since clearly $\delta v_{n} \rightarrow 0$ in $H_{\rho}^{-1}(\mathbb{R} ; H)$, we infer that $\partial_{t, \rho} \mathcal{M} v_{n} \rightarrow y$ in $H_{\rho}^{-1}(\mathbb{R} ; H)$. Moreover, noting that $\partial_{t, \rho}^{-1} v_{n} \rightarrow 0$ in $L_{2, \rho}(\mathbb{R} ; H)$ we derive

$$
\mathcal{M} v_{n}=\mathcal{M} \partial_{t, \rho} \partial_{t, \rho}^{-1} v_{n}=\partial_{t, \rho} \mathcal{M} \partial_{t, \rho}^{-1} v_{n}-\mathcal{M}^{\prime} \partial_{t, \rho}^{-1} v_{n} \rightarrow 0
$$

in $H_{\rho}^{-1}(\mathbb{R} ; H)$ and hence, $\partial_{t, \rho} \mathcal{M} v_{n} \rightarrow 0$ in $H_{\rho}^{-2}(\mathbb{R} ; H)$. This gives $y=0$ and thus, the operator is closable.

Let now $v \in L_{2, \rho}(\mathbb{R} ; H)$. Then we estimate

$$
\begin{aligned}
\operatorname{Re}\left\langle\left(\partial_{t, \rho} \mathcal{M}+\delta\right) v, v\right\rangle_{\rho,-1} & =\operatorname{Re}\left\langle\mathcal{M} v+\partial_{t, \rho}^{-1} \delta v, \partial_{t, \rho}^{-1} v\right\rangle_{\rho} \\
& =\operatorname{Re}\left\langle\mathcal{M} \partial_{t, \rho} \partial_{t, \rho}^{-1} v+\partial_{t, \rho}^{-1} \delta v, \partial_{t, \rho}^{-1} v\right\rangle_{\rho} \\
& =\operatorname{Re}\left\langle\left(\partial_{t, \rho} \mathcal{M}-\mathcal{M}^{\prime}+\delta\right) \partial_{t, \rho}^{-1} v, \partial_{t, \rho}^{-1} v\right\rangle_{\rho} \\
& \geq\left(c+\delta-\left\|\mathcal{M}^{\prime}+\mathcal{N}\right\|\right)\left|\partial_{t, \rho}^{-1} v\right|_{\rho}^{2} \\
& =\left(c+\delta-\left\|\mathcal{M}^{\prime}+\mathcal{N}\right\|\right)|v|_{\rho,-1}^{2},
\end{aligned}
$$

where we have used Lemma 3.4. Since $L_{2, \rho}(\mathbb{R} ; H)$ is a core for $\mathcal{T}_{\delta}$, the $\left(c+\delta-\left\|\mathcal{M}^{\prime}+\mathcal{N}\right\|\right)$ monotonicity follows.

For proving the maximal monotonicity, it suffices to show that $\operatorname{ran}\left(\mathcal{T}_{\delta}\right)$ is dense in $H_{\rho}^{-1}(\mathbb{R} ; H)$. Note that, since $\delta>\|\mathcal{N}\|$, we have that $\partial_{t, \rho} \mathcal{M}+\delta$ is $c$-maximal monotone on $L_{2, \rho}(\mathbb{R} ; H)$ by Lemma 3.4. Thus, in particular $L_{2, \rho}(\mathbb{R} ; H) \subseteq \operatorname{ran}\left(\mathcal{T}_{\delta}\right)$ and hence, the assertion follows.

Definition. We define the set

$$
K:=\left\{\delta>\left\|\mathcal{M}^{\prime}\right\|+\|\mathcal{N}\| ; \forall g \in L_{2, \rho}(\mathbb{R} ; H):\left(\mathcal{T}_{\delta}+\mathcal{B}_{\lambda}\right)^{-1}(g) \in L_{2, \rho}(\mathbb{R} ; H)\right\} .
$$

We aim to prove that $K=]\left\|\mathcal{M}^{\prime}\right\|+\|\mathcal{N}\|, \infty[$. We start with the following observation.

Lemma 3.9. Let $\delta \in K$. Then

$$
\left|\left(\mathcal{T}_{\delta}+\mathcal{B}_{\lambda}\right)^{-1}(g)\right|_{\rho} \leq \frac{1}{c}|g|_{\rho}
$$

for each $g \in L_{2, \rho}(\mathbb{R} ; H)$.

Proof. We set $v:=\left(\mathcal{T}_{\delta}+\mathcal{B}_{\lambda}\right)^{-1}(g) \in L_{2, \rho}(\mathbb{R} ; H)$. Then $\left(\partial_{t, \rho} \mathcal{M}+\delta\right) v=\mathcal{T}_{\delta} v=g-$ $B_{\lambda}(v) \in L_{2, \rho}(\mathbb{R} ; H)$ by Proposition 3.7 and hence, $v \in \operatorname{dom}\left(\partial_{t, \rho} \mathcal{M}\right)$. We then estimate by using Proposition 3.7

$$
\begin{aligned}
\operatorname{Re}\langle g, v\rangle_{\rho} & =\operatorname{Re}\left\langle\mathcal{T}_{\delta} v+B_{\lambda}(v), v\right\rangle_{\rho} \\
& \geq \operatorname{Re}\left\langle\left(\partial_{t, \rho} \mathcal{M}+\delta\right) v, v\right\rangle_{\rho} \\
& \geq c|v|_{\rho}^{2}
\end{aligned}
$$


where we again have used that $\partial_{t, \rho} \mathcal{M}+\delta$ is $c$-maximal monotone on $L_{2, \rho}(\mathbb{R} ; H)$ since $\delta>\|\mathcal{N}\|$. By applying the Cauchy-Schwarz inequality on the left hand side, we derive the desired inequality.

Corollary 3.10. If $K \neq \emptyset$ then $K=]\left\|\mathcal{M}^{\prime}\right\|+\|\mathcal{N}\|, \infty[$.

Proof. We prove that for $\delta \in K$ it follows that $B(\delta, c) \cap]\left\|\mathcal{M}^{\prime}\right\|+\|\mathcal{N}\|, \infty[\subseteq K$. This would yield the assertion. So let $\delta \in K$ and choose $\delta^{\prime}>\left\|\mathcal{M}^{\prime}\right\|+\|\mathcal{N}\|$ with $\left|\delta-\delta^{\prime}\right|<c$. For $g \in L_{2, \rho}(\mathbb{R} ; H)$ we define the sequence $\left(v_{n}\right)_{n \in \mathbb{N}}$ in $L_{2, \rho}(\mathbb{R} ; H)$ recursively by $v_{0}:=0$ and

$$
v_{n+1}:=\left(\mathcal{T}_{\delta}+\mathcal{B}_{\lambda}\right)^{-1}\left(g-\left(\delta^{\prime}-\delta\right) v_{n}\right)
$$

for $n \in \mathbb{N}$. Note that indeed $v_{n+1} \in L_{2, \rho}(\mathbb{R} ; H)$ since $\delta \in K$. Moreover, since $\mid\left(\mathcal{T}_{\delta}+\right.$ $\left.\mathcal{B}_{\lambda}\right)\left.^{-1}\right|_{\operatorname{Lip}, H_{\rho}^{-1}(\mathbb{R} ; H)} \leq \frac{1}{c}$, we infer that the mapping

$$
H_{\rho}^{-1}(\mathbb{R} ; H) \ni w \mapsto\left(\mathcal{T}_{\delta}+\mathcal{B}_{\lambda}\right)^{-1}\left(g-\left(\delta^{\prime}-\delta\right) w\right) \in H_{\rho}^{-1}(\mathbb{R} ; H)
$$

is a strict contraction and thus, it has a unique fixed point $v \in H_{\rho}^{-1}(\mathbb{R} ; H)$. Moreover, $v_{n} \rightarrow v$ in $H_{\rho}^{-1}(\mathbb{R} ; H)$ as $n \rightarrow \infty$. Note, that the fixed point $v$ satisfies

$$
\left(\mathcal{T}_{\delta^{\prime}}+\mathcal{B}_{\lambda}\right)(v)=g
$$

and thus, to complete the proof, we need to show that $v \in L_{2, \rho}(\mathbb{R} ; H)$. For doing so, it suffices to show that $\sup _{n \in \mathbb{N}}\left|v_{n}\right|_{\rho}<\infty$ by Lemma 2.5. Using Lemma 3.9, we estimate

$$
\left|v_{n+1}\right|_{\rho} \leq \frac{1}{c}\left(|g|_{\rho}+\left|\delta^{\prime}-\delta\right|\left|v_{n}\right|_{\rho}\right)
$$

for $n \in \mathbb{N}$ and thus, by induction

$$
\left|v_{n}\right|_{\rho} \leq \frac{1}{c}|g|_{\rho} \sum_{j=0}^{n-1}\left(\frac{\left|\delta^{\prime}-\delta\right|}{c}\right)^{j} \quad(n \in \mathbb{N}) .
$$

As $\left|\delta^{\prime}-\delta\right|<c$, we infer the boundedness of $\left(v_{n}\right)_{n \in \mathbb{N}}$ and hence, the assertion follows.

Proposition 3.11. $K=]\left\|\mathcal{M}^{\prime}\right\|+\|\mathcal{N}\|, \infty[$.

Proof. We choose $\delta>\frac{1}{\lambda}+\|\mathcal{N}\|+\left\|\mathcal{M}^{\prime}\right\|$ and prove that $\delta \in K$. Then the assertion follows by Corollary 3.10. Let $g \in L_{2, \rho}(\mathbb{R} ; H)$ and define the sequence $\left(v_{n}\right)_{n \in \mathbb{N}}$ in $L_{2, \rho}(\mathbb{R} ; H)$ recursively by $v_{0}:=0$ and

$$
v_{n+1}:=\mathcal{T}_{\delta}^{-1}\left(g-B_{\lambda}\left(v_{n}\right)\right)=\left(\partial_{t, \rho} \mathcal{M}+\delta\right)^{-1}\left(g-\mathcal{B}_{\lambda}\left(v_{n}\right)\right) \quad(n \in \mathbb{N}) .
$$

Note that $\mathcal{B}_{\lambda}$ leaves $L_{2, \rho}(\mathbb{R} ; H)$ invariant by Proposition 3.7 and that $\partial_{t, \rho} \mathcal{M}+\delta$ is $c$-maximal monotone on $L_{2, \rho}(\mathbb{R} ; H)$ and thus, $v_{n+1}$ lies indeed in $L_{2, \rho}(\mathbb{R} ; H)$. Indeed, $\partial_{t, \rho} \mathcal{M}+\delta$ is even $(c+\delta-\|\mathcal{N}\|)$-maximal monotone and thus, we estimate

$$
\left|v_{n+1}\right|_{\rho} \leq \frac{1}{c+\delta-\|\mathcal{N}\|}\left(|g|_{\rho}+\left|\mathcal{B}_{\lambda}\left(v_{n}\right)\right|_{\rho}\right) \leq \frac{1}{c+\delta-\|\mathcal{N}\|}\left(|g|_{\rho}+\frac{1}{\lambda}\left|\left(v_{n}\right)\right|_{\rho}\right) \quad(n \in \mathbb{N}),
$$


where we have used Proposition 3.7 in the second inequality. Thus, by induction we can estimate

$$
\left|v_{n}\right|_{\rho} \leq \frac{1}{c+\delta-\|\mathcal{N}\|}|g|_{\rho} \sum_{j=0}^{n-1} \frac{1}{\lambda^{j}(c+\delta-\|\mathcal{N}\|)^{j}} \quad(n \in \mathbb{N})
$$

and thus, by the choice of $\delta$, the sequence $\left(v_{n}\right)_{n \in \mathbb{N}}$ is bounded in $L_{2, \rho}(\mathbb{R} ; H)$. Moreover, since $\mathcal{T}_{\delta}$ is $\left(c+\delta-\left\|\mathcal{M}^{\prime}+\mathcal{N}\right\|\right)$-maximal monotone by Proposition 3.8, we infer by the choice of $\delta$ that $\left|\mathcal{T}_{\delta}\right|_{\operatorname{Lip}, H_{\rho}^{-1}(\mathbb{R} ; H)}<\lambda$ and hence

$$
H_{\rho}^{-1}(\mathbb{R} ; H) \ni w \mapsto \mathcal{T}_{\delta}^{-1}\left(g-\mathcal{B}_{\lambda}(w)\right) \in H_{\rho}^{-1}(\mathbb{R} ; H)
$$

is a strict contraction and hence, has a unique fixed point $v \in H_{\rho}^{-1}(\mathbb{R} ; H)$ which satisfies

$$
\left(\mathcal{T}_{\delta}+\mathcal{B}_{\lambda}\right)(v)=g
$$

Since $v_{n} \rightarrow v$ in $H_{\rho}^{-1}(\mathbb{R} ; H)$, we infer that $v \in L_{2, \rho}(\mathbb{R} ; H)$ by Lemma 2.5 and thus, $\delta \in K$.

We now can come to the

Proof of Theorem [3.6. Let $f \in H_{\rho}^{1}(\mathbb{R} ; H)$ and set

$$
u_{\lambda}:=\left(\partial_{t, \rho} \mathcal{M}-\mathcal{M}^{\prime}+\delta+\mathcal{A}_{\lambda}\right)^{-1}(f) .
$$

Moreover, set

$$
v:=\left(\mathcal{T}_{\delta}+\mathcal{B}_{\lambda}\right)^{-1}\left(\partial_{t, \rho} f\right) .
$$

We note that $v \in L_{2, \rho}(\mathbb{R} ; H)$ by Proposition 3.11 and that

$$
|v|_{\rho} \leq \frac{1}{c}\left|\partial_{t, \rho} f\right|_{\rho}=\frac{1}{c}|f|_{\rho, 1}
$$

by Lemma 3.9, We compute

$$
\begin{aligned}
\partial_{t, \rho} \mathcal{M} \partial_{t, \rho}^{-1} v-\mathcal{M}^{\prime} \partial_{t, \rho}^{-1} v+\delta \partial_{t, \rho}^{-1} v & =\mathcal{M} v+\delta \partial_{t, \rho}^{-1} v \\
& =\partial_{t, \rho}^{-1} \mathcal{T}_{\delta} v \\
& =\partial_{t, \rho}^{-1}\left(\partial_{t, \rho} f-\mathcal{B}_{\lambda}(v)\right) \\
& =f-\mathcal{A}_{\lambda}\left(\partial_{t, \rho}^{-1} v\right)
\end{aligned}
$$

and hence,

$$
\left(\partial_{t, \rho} \mathcal{M}-\mathcal{M}^{\prime}+\delta+\mathcal{A}_{\lambda}\right)\left(\partial_{t, \rho}^{-1} v\right)=f,
$$

which shows $u_{\lambda}=\partial_{t, \rho}^{-1} v \in H_{\rho}^{1}(\mathbb{R} ; H)$. Moreover,

$$
\left|u_{\lambda}\right|_{\rho, 1}=|v|_{\rho} \leq \frac{1}{c}|f|_{\rho, 1} .
$$


Corollary 3.12. Let $\delta>\left\|\mathcal{M}^{\prime}\right\|+\|\mathcal{N}\|$. Then $\overline{\partial_{t, \rho} \mathcal{M}-\mathcal{M}^{\prime}+\delta+\mathcal{A}}$ is c-maximal monotone and hence,

$$
\mathcal{S}_{\rho, \text { aux }}:=\left(\overline{\partial_{t, \rho} \mathcal{M}-\mathcal{M}^{\prime}+\delta+\mathcal{A}}\right)^{-1}: L_{2, \rho}(\mathbb{R} ; H) \rightarrow L_{2, \rho}(\mathbb{R} ; H)
$$

is Lipschitz-continuous. Moreover, for $f \in H_{\rho}^{1}(\mathbb{R} ; H)$ we have that $\mathcal{S}_{\rho, \text { aux }}(f) \in H_{\rho}^{1}(\mathbb{R} ; H) \cap$ $\operatorname{dom}\left(\left(\partial_{t, \rho} \mathcal{M}-\mathcal{M}^{\prime}+\delta+\mathcal{A}\right)^{-1}\right)$.

Proof. We already know that $\overline{\partial_{t, \rho} \mathcal{M}-\mathcal{M}^{\prime}+\delta+\mathcal{A}}$ is $c$-monotone by Proposition 3.5 . For proving the maximal monotonicity it suffices to show that there is a dense subset $D \subseteq L_{2, \rho}(\mathbb{R} ; H)$ such that for each $f \in D$ there exists $u \in L_{2, \rho}(\mathbb{R} ; H)$ with

$$
(u, f) \in \overline{\partial_{t, \rho} \mathcal{M}-\mathcal{M}^{\prime}+\delta+\mathcal{A}} .
$$

We show that the latter is true for $D=H_{\rho}^{1}(\mathbb{R} ; H)$. So, let $f \in H_{\rho}^{1}(\mathbb{R} ; H)$. For showing that a solution $u \in L_{2, \rho}(\mathbb{R} ; H)$ exists, it suffices to prove that $\sup _{\lambda>0}\left|\mathcal{A}_{\lambda}\left(u_{\lambda}\right)\right|_{\rho}<\infty$ for

$$
u_{\lambda}:=\left(\partial_{t, \rho} \mathcal{M}-\mathcal{M}^{\prime}+\delta+\mathcal{A}_{\lambda}\right)^{-1}(f)
$$

by Proposition 2.14. According to Theorem 3.6 we know that $u_{\lambda} \in H_{\rho}^{1}(\mathbb{R} ; H)$ and that

$$
\left|u_{\lambda}\right|_{\rho, 1} \leq \frac{1}{c}|f|_{\rho, 1}
$$

for each $\lambda>0$. Thus, we can estimate

$$
\begin{aligned}
\left|\mathcal{A}_{\lambda}\left(u_{\lambda}\right)\right|_{\rho} & =\left|f-\left(\partial_{t, \rho} \mathcal{M}-\mathcal{M}^{\prime}\right) u_{\lambda}-\delta u_{\lambda}\right|_{\rho} \\
& =\left|f-\mathcal{M} \partial_{t, \rho} u_{\lambda}-\delta u_{\lambda}\right|_{\rho} \\
& \leq|f|_{\rho}+\|\mathcal{M}\|\left|u_{\lambda}\right|_{\rho, 1}+\frac{\delta}{\rho}\left|u_{\lambda}\right|_{\rho, 1} \\
& \leq|f|_{\rho}+\frac{1}{c}\left(\|\mathcal{M}\|+\frac{\delta}{\rho}\right)|f|_{\rho, 1} .
\end{aligned}
$$

Summarising, we have shown the $c$-maximal monotonicity of $\overline{\partial_{t, \rho} \mathcal{M}-\mathcal{M}^{\prime}+\delta+\mathcal{A}}$. Moreover, $u_{\lambda} \rightarrow u$ as $\lambda \rightarrow 0+$ in $L_{2, \rho}(\mathbb{R} ; H)$ and

$$
(u, f) \in \partial_{t, \rho} \mathcal{M}-\mathcal{M}^{\prime}+\delta+\mathcal{A}
$$

by Proposition 2.14, i.e. $u=\mathcal{S}_{\rho, \operatorname{aux}}(f) \in \operatorname{dom}\left(\left(\partial_{t, \rho} \mathcal{M}-\mathcal{M}^{\prime}+\delta+\mathcal{A}\right)^{-1}\right)$. Moreover, since $\left(u_{\lambda}\right)_{\lambda>0}$ is bounded in $H_{\rho}^{1}(\mathbb{R} ; H)$, we infer that also $u=\mathcal{S}_{\rho, \text { aux }}(f) \in H_{\rho}^{1}(\mathbb{R} ; H)$ by Lemma 2.5.

With this result at hand, we can prove our main theorem.

Proof of Theorem 3.1. We have that $\overline{\partial_{t, \rho} \mathcal{M}+\mathcal{N}+\mathcal{A}}$ is $c$-monotone by Proposition 3.3 and hence,

$$
\overline{\partial_{t, \rho} \mathcal{M}+\mathcal{N}+\mathcal{A}}-c=\left(\overline{\partial_{t, \rho} \mathcal{M}-\mathcal{M}^{\prime}+\delta+\mathcal{A}}-c\right)+\left(\mathcal{M}^{\prime}+\mathcal{N}-\delta\right)
$$

is maximal monotone by Proposition 2.12 and Corollary 3.12 . 


\section{Causality and independence of $\rho$}

The aim of this section is twofold. First, we prove that the solution operator $\mathcal{S}_{\rho}$ associated with the differential inclusion

$$
(u, f) \in \overline{\partial_{t, \rho} \mathcal{M}+\mathcal{N}+\mathcal{A}}
$$

is causal under suitable additional conditions on $\mathcal{M}, \mathcal{N}$ and $\mathcal{A}$. And second, we prove that the solution operator is independent of the choice of the parameter $\rho$, if $\mathcal{M}, \mathcal{N}$ and $\mathcal{A}$ are independent of $\rho$ in some sense.

We start with the causality and define, what we mean by a causal operator.

Definition. Let $F: L_{2, \rho}(\mathbb{R} ; H) \rightarrow L_{2, \rho}(\mathbb{R} ; H)$. Then, $F$ is called causal, if for each $f, g \in L_{2, \rho}(\mathbb{R} ; H)$ when $f=g$ on $\left.]-\infty, a\right]$ for some $a \in \mathbb{R}$, also $F(f)=F(g)$ on $\left.]-\infty, a\right]$ holds.

Remark 4.1. (a) Causality is a crucial property for operators describing a temporal evolution, as it says that the solution $u$ up to some time $a \in \mathbb{R}$ does not depend on the behaviour of the right hand side $f$ on $[a, \infty[$.

(b) For mappings not defined on the whole space $L_{2, \rho}(\mathbb{R} ; H)$ one has to adopt the definition of causality in a way, that it is preserved under closure (i.e. $\bar{F}$ is causal if $F$ is causal) and that it coincides with the causality defined above if $F$ is defined on the whole $L_{2, \rho}(\mathbb{R} ; H)$. This was done in [33].

We now state the hypotheses which allow us to deduce the causality of the solution operator.

Hypotheses B. Let $\mathcal{M}, \mathcal{N}, \mathcal{M}^{\prime} \in L\left(L_{2, \rho}(\mathbb{R} ; H)\right)$ be such that:

(a) $\mathcal{M} \partial_{t, \rho} \subseteq \partial_{t, \rho} \mathcal{M}-\mathcal{M}^{\prime}$

(b) There exists $c>0$ such that

$$
\operatorname{Re}\left\langle\left(\partial_{t, \rho} \mathcal{M}+\mathcal{N}\right) \varphi, \chi_{\mathbb{R}_{\leq a}} \varphi\right\rangle_{\rho} \geq c\left|\chi_{\mathbb{R}_{\leq a}} \varphi\right|_{\rho}^{2}
$$

for each $\varphi \in C_{c}^{\infty}(\mathbb{R} ; H)$ and each $a \in \mathbb{R}$.

Moreover, let $\mathcal{A} \subseteq L_{2, \rho}(\mathbb{R} ; H) \times L_{2, \rho}(\mathbb{R} ; H)$ be maximal monotone such that

$$
\forall u, v \in L_{2, \rho}(\mathbb{R} ; H):\left((u, v) \in \mathcal{A} \Rightarrow \forall h \geq 0:\left(\tau_{h} u, \tau_{h} v\right) \in \mathcal{A}\right)
$$

and assume that $\chi_{\mathbb{R}_{\leq a}} \mathcal{A}$ is monotone for each $a \in \mathbb{R}$.

Remark 4.2. We note that Hypotheses B imply Hypotheses A since (b) of Hypotheses A follows from Hypotheses $\mathrm{B}$ (b) by letting $a$ tend to infinity. Moreover, the monotonicity of $\chi_{\mathbb{R}_{\leq a}} \mathcal{A}$ states that for each $(u, v),(x, y) \in \mathcal{A}$ we have that

$$
\operatorname{Re}\left\langle u-x, \chi_{\mathbb{R}_{\leq a}}(v-y)\right\rangle_{\rho}=\operatorname{Re}\left\langle\chi_{\mathbb{R}_{\leq a}}(u-x), v-y\right\rangle_{\rho} \geq 0 .
$$


Lemma 4.3. Assume Hypotheses B. Then

$$
\operatorname{Re}\left\langle\left(\partial_{t, \rho} \mathcal{M}+\mathcal{N}\right) u, \chi_{\mathbb{R}_{\leq a}} u\right\rangle_{\rho} \geq c\left|\chi_{\mathbb{R}_{\leq a}} u\right|_{\rho}^{2}
$$

for each $u \in \operatorname{dom}\left(\partial_{t, \rho} \mathcal{M}\right)$ and $a \in \mathbb{R}$.

Proof. By Proposition 3.3, $C_{c}^{\infty}(\mathbb{R} ; H)$ is a core for $\partial_{t, \rho} \mathcal{M}$. Hence, the assertion follows by approximating $u$ with elements in $C_{c}^{\infty}(\mathbb{R} ; H)$ and noting that multiplication with $\chi_{\mathbb{R}_{\leq a}}$ is continuous on $L_{2, \rho}(\mathbb{R} ; H)$.

Theorem 4.4 (Causality). Assume Hypotheses $B$. Then the solution operator $\mathcal{S}_{\rho}:=$ $\left(\overline{\partial_{t, \rho} \mathcal{M}+\mathcal{N}+\mathcal{A}}\right)^{-1}$ from Theorem 3.1 is causal.

Proof. Let $f, g \in L_{2, \rho}(\mathbb{R} ; H)$ and assume that $f=g$ on $\left.]-\infty, a\right]$ for some $a \in \mathbb{R}$. We set

$$
u:=\mathcal{S}_{\rho}(f) \text { and } v:=\mathcal{S}_{\rho}(g) .
$$

Since $\mathcal{S}_{\rho}=\left(\overline{\partial_{t, \rho} \mathcal{M}+\mathcal{N}+\mathcal{A}}\right)^{-1}=\overline{\left(\partial_{t, \rho} \mathcal{M}+\mathcal{N}+\mathcal{A}\right)^{-1}}$ is defined on the whole $L_{2, \rho}(\mathbb{R} ; H)$ it follows that $\operatorname{dom}\left(\left(\partial_{t, \rho} \mathcal{M}+\mathcal{N}+\mathcal{A}\right)^{-1}\right)$ is dense in $L_{2, \rho}(\mathbb{R} ; H)$. Hence, there exist sequences $\left(f_{n}\right)_{n \in \mathbb{N}}$ and $\left(g_{n}\right)_{n \in \mathbb{N}}$ in $\operatorname{dom}\left(\left(\partial_{t, \rho} \mathcal{M}+\mathcal{N}+\mathcal{A}\right)^{-1}\right)$ such that $f_{n} \rightarrow f$ and $g_{n} \rightarrow g$ in $L_{2, \rho}(\mathbb{R} ; H)$. Setting

$$
u_{n}:=\mathcal{S}_{\rho}\left(f_{n}\right) \text { and } v_{n}:=\mathcal{S}_{\rho}\left(g_{n}\right)
$$

for $n \in \mathbb{N}$, it follows that $u_{n} \rightarrow u$ and $v_{n} \rightarrow v$ in $L_{2, \rho}(\mathbb{R} ; H)$ by continuity of $\mathcal{S}_{\rho}$. By definition, for $n \in \mathbb{N}$ there exist $x_{n}, y_{n} \in L_{2, \rho}(\mathbb{R} ; H)$ such that $\left(u_{n}, x_{n}\right),\left(v_{n}, y_{n}\right) \in \mathcal{A}$ and

$$
\begin{aligned}
\partial_{t, \rho} \mathcal{M} u_{n}+\mathcal{N} u_{n}+x_{n} & =f_{n} \\
\partial_{t, \rho} \mathcal{M} v_{n}+\mathcal{N} v_{n}+y_{n} & =g_{n} .
\end{aligned}
$$

Moreover, by Lemma 4.3 and the monotonicity of $\chi_{\mathbb{R}_{\leq a}} \mathcal{A}$ we estimate

$$
\begin{aligned}
& \operatorname{Re}\left\langle f_{n}-g_{n}, \chi_{\mathbb{R}_{\leq a}}\left(u_{n}-v_{n}\right)\right\rangle_{\rho} \\
& =\operatorname{Re}\left\langle\left(\partial_{t, \rho} \mathcal{M}+\mathcal{N}\right)\left(u_{n}-v_{n}\right), \chi_{\mathbb{R}_{\leq a}}\left(u_{n}-v_{n}\right)\right\rangle_{\rho}+\operatorname{Re}\left\langle x_{n}-y_{n}, \chi_{\mathbb{R}_{\leq a}}\left(u_{n}-v_{n}\right)\right\rangle_{\rho} \\
& \geq c\left|\chi_{\mathbb{R}_{\leq a}}\left(u_{n}-v_{n}\right)\right|_{\rho}^{2} .
\end{aligned}
$$

Letting $n$ tend to infinity, it follows that

$$
0=\operatorname{Re}\left\langle f-g, \chi_{\mathbb{R}_{\leq a}}(u-v)\right\rangle_{\rho} \geq c\left|\chi_{\mathbb{R}_{\leq a}}(u-v)\right|_{\rho}^{2},
$$

which proves $u=v$ on $]-\infty, a]$ and hence, the causality of $\mathcal{S}_{\rho}$ follows.

In order to formulate the independence result we need the concept of evolutionary mappings. These mappings were introduced in [32, 35], however in a more general way than needed here. 
Definition. Let $\rho_{0}>0$. A linear mapping $T: C_{c}^{\infty}(\mathbb{R} ; H) \rightarrow \bigcap_{\mu \geq \rho_{0}} L_{2, \mu}(\mathbb{R} ; H)$ is called evolutionary at $\rho_{0}$, if for each $\rho \geq \rho_{0}$ the mapping

$$
T: C_{c}^{\infty}(\mathbb{R} ; H) \subseteq L_{2, \rho}(\mathbb{R} ; H) \rightarrow L_{2, \rho}(\mathbb{R} ; H)
$$

is bounded and $\|T\|_{\mathrm{ev}, \rho_{0}}:=\sup _{\rho \geq \rho_{0}}\|T\|_{L\left(L_{2, \rho}(\mathbb{R} ; H)\right)}<\infty$. If $T$ is evolutionary at $\rho_{0}$, we denote the (unique) extension of $T$ to $L_{2, \rho}(\mathbb{R} ; H)$ by $T_{\rho}$ for each $\rho \geq \rho_{0}$.

We are now ready to formulate our hypotheses, which will allow us to prove the independence of the parameter $\rho$.

Hypotheses C. Let $\rho_{0}>0$ and $\mathcal{M}, \mathcal{N}, \mathcal{M}^{\prime}$ evolutionary at $\rho_{0}$ such that

(a) $\forall \varphi \in C_{c}^{\infty}(\mathbb{R} ; H): \mathcal{M} \varphi^{\prime}+\mathcal{M}^{\prime} \varphi=(\mathcal{M} \varphi)^{\prime}$ in the sense of distributions.

(b) There exists $c>0$ such that for all $\rho \geq \rho_{0}$

$$
\operatorname{Re}\left\langle\left(\partial_{t, \rho} \mathcal{M}+\mathcal{N}\right) \varphi, \chi_{\mathbb{R}_{\leq a}} \varphi\right\rangle_{\rho} \geq c\left|\chi_{\mathbb{R}_{\leq a}} \varphi\right|_{\rho}^{2}
$$

for each $\varphi \in C_{c}^{\infty}(\mathbb{R} ; H)$ and each $a \in \mathbb{R}$.

Moreover, let $A \subseteq H \times H$ be maximal monotone with $(0,0) \in A$.

Remark 4.5. We note that by Lemma 2.7 the extension of $A$ to $L_{2, \rho}(\mathbb{R} ; H)$ is maximal monotone for each $\rho \in \mathbb{R}$. We will denote this extension by $\mathcal{A}_{\rho}$.

First, we show that Hypotheses $\mathrm{C}$ imply Hypotheses $\mathrm{B}$ for the operators $\mathcal{M}_{\rho}, \mathcal{N}_{\rho}, \mathcal{M}_{\rho}^{\prime}$ and the relation $\mathcal{A}_{\rho}$ for each $\rho \geq \rho_{0}$.

Lemma 4.6. We assume Hypotheses $\square$. Then for each $\rho \geq \rho_{0}$ the operators $\mathcal{M}_{\rho}, \mathcal{N}_{\rho}, \mathcal{M}_{\rho}^{\prime}$ and the relation $\mathcal{A}_{\rho}$ satisfy Hypotheses B. Moreover, the constant $c$ in Hypotheses B (b) is independent of $\rho$.

Proof. It is clear that $\mathcal{M}_{\rho}, \mathcal{N}_{\rho}, \mathcal{M}_{\rho}^{\prime}$ satisfy Hypotheses B (b). Moreover, the constant $c$ in Hypotheses B (b) is the same as in Hypotheses $\mathrm{C}(\mathrm{b})$ and thus, independent of $\rho$. To show Hypotheses $\mathrm{B}(\mathrm{a})$, let $u \in \operatorname{dom}\left(\partial_{t, \rho}\right)$. We need to show that $\mathcal{M}_{\rho} u \in \operatorname{dom}\left(\partial_{t, \rho}\right)$ and

$$
\partial_{t, \rho} \mathcal{M}_{\rho} u=\mathcal{M}_{\rho} \partial_{t, \rho} u+\mathcal{M}_{\rho}^{\prime} u
$$

Choose a sequence $\left(\varphi_{n}\right)_{n \in \mathbb{N}}$ in $C_{c}^{\infty}(\mathbb{R} ; H)$ such that $\varphi_{n} \rightarrow u$ in $H_{\rho}^{1}(\mathbb{R} ; H)$. Note that by Hypotheses C (a) we have that

$$
\left(\mathcal{M} \varphi_{n}\right)^{\prime}=\mathcal{M} \varphi_{n}^{\prime}+\mathcal{M}^{\prime} \varphi_{n} \in L_{2, \rho}(\mathbb{R} ; H) \quad(n \in \mathbb{N})
$$

and hence, $\mathcal{M} \varphi_{n} \in \operatorname{dom}\left(\partial_{t, \rho}\right)$ (compare Remark 2.1 (a)). Moreover,

$$
\partial_{t, \rho} \mathcal{M} \varphi_{n}=\mathcal{M} \varphi_{n}^{\prime}+\mathcal{M}^{\prime} \varphi_{n} \rightarrow \mathcal{M}_{\rho} \partial_{t, \rho} u+\mathcal{M}_{\rho}^{\prime} u \quad(n \rightarrow \infty)
$$


in $L_{2, \rho}(\mathbb{R} ; H)$ and since also $\mathcal{M} \varphi_{n} \rightarrow \mathcal{M}_{\rho} u$, the assertion follows by the closedness of $\partial_{t, \rho}$. It is left to prove that $\mathcal{A}_{\rho}$ satisfies Hypotheses C. We had already remarked that $\mathcal{A}_{\rho}$ is maximal monotone by Lemma 2.7 Moreover, for $(u, v) \in \mathcal{A}_{\rho}$ and $h \geq 0$ we have that

$$
\begin{aligned}
(u, v) \in \mathcal{A}_{\rho} & \Rightarrow(u(t), v(t)) \in A \quad(t \in \mathbb{R} \text { a.e. }) \\
& \Rightarrow(u(t+h), v(t+h)) \in A \quad(t \in \mathbb{R} \text { a.e. }) \\
& \Rightarrow\left(\tau_{h} u, \tau_{h} v\right) \in \mathcal{A}_{\rho} .
\end{aligned}
$$

Moreover, for $a \in \mathbb{R}$ and $(u, v),(x, y) \in \mathcal{A}_{\rho}$ we have that

$$
\operatorname{Re}\left\langle u-x, \chi_{\mathbb{R}_{\leq a}}(v-y)\right\rangle_{\rho}=\int_{-\infty}^{a} \operatorname{Re}\langle u(t)-x(t), v(t)-y(t)\rangle \mathrm{e}^{-2 \rho t} \mathrm{~d} t \geq 0,
$$

since $(u(t), v(t)),(x(t), y(t)) \in A$ for almost every $t \in \mathbb{R}$ and $A$ is monotone.

In order to prove that the solution operator $\mathcal{S}_{\rho}=\left(\overline{\partial_{t, \rho} \mathcal{M}_{\rho}+\mathcal{N}_{\rho}+\mathcal{A}_{\rho}}\right)^{-1}$ is independent of $\rho$ in the sense that for each $\mu, \rho \geq \rho_{0}$

$$
\mathcal{S}_{\rho}(f)=\mathcal{S}_{\mu}(f) \quad\left(f \in L_{2, \rho}(\mathbb{R} ; H) \cap L_{2, \mu}(\mathbb{R} ; H)\right)
$$

we prove this property for the solution operator $\mathcal{S}_{\rho, \text { aux }}=\left(\overline{\partial_{t, \rho} \mathcal{M}_{\rho}-\mathcal{M}_{\rho}^{\prime}+\delta+\mathcal{A}_{\rho}}\right)^{-1}$ with $\delta>\left\|\mathcal{M}^{\prime}\right\|_{\mathrm{ev}, \rho_{0}}+\|\mathcal{N}\|_{\mathrm{ev}, \rho_{0}}$ first. For doing so, we need to show that the solution operator $\mathcal{S}_{\rho \text {,aux }}$ is causal. This will be shown in the next proposition. However, we first need the following lemma, showing an important property of evolutionary mappings.

Lemma 4.7. Let $\rho_{0}>0$ and $T: C_{c}^{\infty}(\mathbb{R} ; H) \rightarrow \bigcap_{\mu \geq \rho_{0}} L_{2, \mu}(\mathbb{R} ; H)$ evolutionary at $\rho_{0}$. Then, for each $\mu, \rho \geq \rho_{0}$ and $f \in L_{2, \rho}(\mathbb{R} ; H) \cap L_{2, \mu}(\mathbb{R} ; H)$ we have that

$$
T_{\mu} f=T_{\rho} f .
$$

Moreover, for each $\rho \geq \rho_{0}$ the mapping $T_{\rho}$ is causal.

Proof. The first assertion is clear, since a function $f \in L_{2, \rho}(\mathbb{R} ; H) \cap L_{2, \mu}(\mathbb{R} ; H)$ can be approximated by one sequence $\left(\varphi_{n}\right)_{n \in \mathbb{N}}$ in $C_{c}^{\infty}(\mathbb{R} ; H)$ such that $\varphi_{n} \rightarrow f$ in $L_{2, \mu}(\mathbb{R} ; H)$ and $L_{2, \rho}(\mathbb{R} ; H)$, see e.g. [28, Lemma 3.5]. For proving the causality of $T_{\rho}$ we note that due to linearity it suffices to show for $u \in L_{2, \rho}(\mathbb{R} ; H)$ with spt $u \subseteq \mathbb{R}_{\geq a}$ for some $a \in \mathbb{R}$ it follows that $\operatorname{spt} T_{\rho} u \subseteq \mathbb{R}_{\geq a}$. So, let $u \in L_{2, \rho}(\mathbb{R} ; H)$ with spt $u \subseteq \mathbb{R}_{\geq a}$ for some $a \in \mathbb{R}$. 
Then $u \in \bigcap_{\mu \geq \rho} L_{2, \mu}(\mathbb{R} ; H)$. For $\mu \geq \rho$ we compute

$$
\begin{aligned}
\int_{-\infty}^{a}\left|\left(T_{\rho} u\right)(t)\right| \mathrm{d} t & =\int_{-\infty}^{a}\left|\left(T_{\mu} u\right)(t)\right| \mathrm{e}^{-\mu t} \mathrm{e}^{\mu t} \mathrm{~d} t \\
& \leq\left|T_{\mu} u\right|_{\mu} \frac{1}{\sqrt{2 \mu}} \mathrm{e}^{\mu a} \\
& \leq \frac{1}{\sqrt{2 \mu}}|T|_{\mathrm{ev}, \rho_{0}}|u|_{\mu} \mathrm{e}^{\mu a} \\
& =\frac{1}{\sqrt{2 \mu}}|T|_{\mathrm{ev}, \rho_{0}}\left(\int_{a}^{\infty}|u(t)|^{2} \mathrm{e}^{-2 \mu t} \mathrm{~d} t\right)^{\frac{1}{2}} \mathrm{e}^{\mu a} \\
& =\frac{1}{\sqrt{2 \mu}}|T|_{\mathrm{ev}, \rho_{0}}\left(\int_{a}^{\infty}|u(t)|^{2} \mathrm{e}^{-2 \rho t} \mathrm{e}^{2(\rho-\mu) t} \mathrm{~d} t\right)^{\frac{1}{2}} \mathrm{e}^{\mu a} \\
& \leq \frac{1}{\sqrt{2 \mu}}|T|_{\mathrm{ev}, \rho_{0}}|u|_{\rho} \mathrm{e}^{\rho a} .
\end{aligned}
$$

Letting $\mu \rightarrow \infty$, we infer that $T_{\rho} u=0$ on $\left.]-\infty, a\right]$ and hence, $\operatorname{spt} T_{\rho} u \subseteq \mathbb{R}_{\geq a}$.

Proposition 4.8. Assume Hypotheses $\square$ and let $\delta>\left\|\mathcal{M}^{\prime}\right\|_{\mathrm{ev}, \rho_{0}}+\|\mathcal{N}\|_{\mathrm{ev}, \rho_{0}}$. Then, for each $\rho \geq \rho_{0}$ the solution operator $\mathcal{S}_{\rho, \text { aux }}$ is causal.

Proof. Let $\rho \geq \rho_{0}$. By Theorem 4.4 it suffices to prove that Hypotheses B hold if we replace $\mathcal{N}_{\rho}$ by $\delta-\mathcal{M}_{\rho}^{\prime}$. Note that only Hypotheses $\mathrm{B}$ (b) has to be verified for $\mathcal{N}$ replaced by $\delta-\mathcal{M}^{\prime}$. So, let $\varphi \in C_{c}^{\infty}(\mathbb{R} ; H)$ and $a \in \mathbb{R}$. Note that by Lemma 4.6 we have that

$$
\operatorname{Re}\left\langle\left(\partial_{t} \mathcal{M}+\mathcal{N}\right) \varphi, \chi_{\mathbb{R}_{\leq a}} \varphi\right\rangle_{\rho} \geq c\left|\chi_{\mathbb{R}_{\leq a}} \varphi\right|_{\rho}^{2}
$$

Moreover, we note that by Lemma 4.7 we have

$$
\chi_{\mathbb{R}_{\leq a}}\left(\mathcal{M}^{\prime}+\mathcal{N}\right) \varphi=\chi_{\mathbb{R}_{\leq a}}\left(\mathcal{M}^{\prime}+\mathcal{N}\right) \chi_{\mathbb{R}_{\leq a}} \varphi .
$$

Hence, we can estimate

$$
\begin{aligned}
\operatorname{Re}\left\langle\left(\partial_{t} \mathcal{M}-\mathcal{M}^{\prime}+\delta\right) \varphi, \chi_{\mathbb{R}_{\leq a}} \varphi\right\rangle_{\rho} & =\operatorname{Re}\left\langle\left(\partial_{t} \mathcal{M}+\mathcal{N}\right) \varphi, \chi_{\mathbb{R}_{\leq a}} \varphi\right\rangle_{\rho}+\operatorname{Re}\left\langle\delta \varphi-\left(\mathcal{M}^{\prime}+\mathcal{N}\right) \varphi, \chi_{\mathbb{R}_{\leq a}} \varphi\right\rangle_{\rho} \\
& \geq c\left|\chi_{\mathbb{R}_{\leq a}} \varphi\right|_{\rho}^{2}+\delta\left|\chi_{\mathbb{R}_{\leq a}} \varphi\right|_{\rho}^{2}-\operatorname{Re}\left\langle\chi_{\mathbb{R}_{\leq a}}\left(\mathcal{M}^{\prime}+\mathcal{N}\right) \varphi, \chi_{\mathbb{R}_{\leq a}} \varphi\right\rangle_{\rho} \\
& =c\left|\chi_{\mathbb{R}_{\leq a}} \varphi\right|_{\rho}^{2}+\left.\left.\delta\right|_{\mathbb{R}_{\leq a}} \varphi\right|_{\rho} ^{2}-\operatorname{Re}\left\langle\left(\mathcal{M}^{\prime}+\mathcal{N}\right) \chi_{\mathbb{R}_{\leq a}} \varphi, \chi_{\mathbb{R}_{\leq a}} \varphi\right\rangle_{\rho} \\
& \geq\left(c+\delta-\left(\left\|\mathcal{M}^{\prime}\right\|_{\mathrm{ev}, \rho_{0}}+\|\mathcal{N}\|_{\mathrm{ev}, \rho_{0}}\right)\right)\left|\chi_{\mathbb{R}_{\leq a}} \varphi\right|_{\rho}^{2} \\
& \geq c\left|\chi_{\mathbb{R}_{\leq a}} \varphi\right|_{\rho}^{2} .
\end{aligned}
$$

With this result at hand, we are able to prove the independence on the parameter $\rho$ for the solution operator $\mathcal{S}_{\rho \text {,aux }}$. 
Proposition 4.9. Assume Hypotheses $\square$ and let $\delta>\left\|\mathcal{M}^{\prime}\right\|_{\mathrm{ev}, \rho_{0}}+\|\mathcal{N}\|_{\mathrm{ev}, \rho_{0}}$. Then, for each $\mu, \rho \geq \rho_{0}$ and each $f \in L_{2, \rho}(\mathbb{R} ; H) \cap L_{2, \mu}(\mathbb{R} ; H)$ we have that

$$
\mathcal{S}_{\rho, \text { aux }}(f)=\mathcal{S}_{\mu, \text { aux }}(f)
$$

as functions in $L_{1, \text { loc }}(\mathbb{R} ; H)$.

Proof. We begin to prove $\mathcal{S}_{\rho \text {,aux }}(f)=\mathcal{S}_{\rho_{0} \text {,aux }}(f)$ for all $\rho \geq \rho_{0}$ and $f \in C_{c}^{\infty}(\mathbb{R} ; H)$. We set

$$
u_{\rho_{0}}:=\mathcal{S}_{\rho_{0}}(f)=\left(\partial_{0, \rho_{0}} \mathcal{M}_{\rho_{0}}-\mathcal{M}_{\rho_{0}}^{\prime}+\delta+\mathcal{A}_{\rho_{0}}\right)^{-1}(f)
$$

where we have used Corollary 3.12, Let $a \in \mathbb{R}$ such that spt $f \subseteq\left[a, \infty\left[\right.\right.$. As $\mathcal{S}_{\rho_{0} \text {,aux }}(0)=0$, since $(0,0) \in \mathcal{A}_{\rho_{0}}$, we derive form the causality of $\mathcal{S}_{\rho_{0} \text {,aux }}$ (see Proposition 4.8) that spt $u_{\rho_{0}} \subseteq\left[a, \infty\left[\right.\right.$ and hence $u_{\rho_{0}} \in \bigcap_{\mu \geq \rho_{0}} L_{2, \mu}(\mathbb{R} ; H)$. Moreover, by Lemma 4.7 and the definition of $\partial_{t, \rho_{0}}, \partial_{t, \rho_{0}} \mathcal{M}_{\rho_{0}} u_{\rho_{0}} \in L_{2, \rho_{0}}(\mathbb{R} ; H)$ is supported on $[a, \infty[$ as well and hence, $\partial_{t, \rho_{0}} \mathcal{M}_{\rho_{0}} u_{\rho_{0}} \in L_{2, \rho}(\mathbb{R} ; H)$. The latter gives $\partial_{t, \rho}^{-1} \partial_{t, \rho_{0}} \mathcal{M}_{\rho_{0}} u_{\rho_{0}}=\mathcal{M}_{\rho_{0}} u_{\rho_{0}}=\mathcal{M}_{\rho} u_{\rho_{0}}$ and hence, $\mathcal{M}_{\rho} u_{\rho_{0}} \in \operatorname{dom}\left(\partial_{t, \rho}\right)$ with $\partial_{t, \rho} \mathcal{M}_{\rho} u_{\rho_{0}}=\partial_{t, \rho_{0}} \mathcal{M}_{\rho_{0}} u_{\rho_{0}}$. Consequently,

$$
\left(\partial_{0, \rho_{0}} \mathcal{M}_{\rho_{0}}-\mathcal{M}_{\rho_{0}}^{\prime}+\delta\right) u_{\rho_{0}}=\left(\partial_{0, \rho} \mathcal{M}_{\rho}-\mathcal{M}_{\rho}^{\prime}+\delta\right) u_{\rho_{0}} \in L_{2, \rho}(\mathbb{R} ; H)
$$

and thus,

$$
\left(u_{\rho_{0}}, f-\left(\partial_{0, \rho} \mathcal{M}_{\rho}-\mathcal{M}_{\rho}^{\prime}+\delta\right) u_{\rho_{0}}\right) \in \mathcal{A}_{\rho}
$$

The latter gives that $u_{\rho_{0}}$ satisfies

$$
\left(u_{\rho_{0}}, f\right) \in \partial_{0, \rho} \mathcal{M}_{\rho}-\mathcal{M}_{\rho}^{\prime}+\delta+\mathcal{A}_{\rho}
$$

and hence $\mathcal{S}_{\rho_{0} \text {,aux }}(f)=u_{\rho_{0}}=\mathcal{S}_{\rho}(f)$.

Let now $f \in L_{2, \rho}(\mathbb{R} ; H) \cap L_{2, \mu}(\mathbb{R} ; H)$. Then there exists a sequence $\left(f_{n}\right)_{n \in \mathbb{N}}$ in $C_{c}^{\infty}(\mathbb{R} ; H)$ with $f_{n} \rightarrow f$ in $L_{2, \rho}(\mathbb{R} ; H)$ and $L_{2, \mu}(\mathbb{R} ; H)$ (see e.g. [28, Lemma 3.5]). By what we have shown above, we infer

$$
\mathcal{S}_{\rho, \text { aux }}(f)=\lim _{n \rightarrow \infty} \mathcal{S}_{\rho, \text { aux }}\left(f_{n}\right)=\lim _{n \rightarrow \infty} \mathcal{S}_{\mu, \text { aux }}\left(f_{n}\right)=\mathcal{S}_{\mu, \text { aux }}(f) .
$$

Theorem 4.10 (Independence of the parameter $\rho$ ). Assume Hypotheses $\square$. Then, for each $\mu, \rho \geq \rho_{0}$ and each $f \in L_{2, \rho}(\mathbb{R} ; H) \cap L_{2, \mu}(\mathbb{R} ; H)$ we have that

$$
\mathcal{S}_{\rho}(f)=\mathcal{S}_{\mu}(f)
$$

as functions in $L_{1, \text { loc }}(\mathbb{R} ; H)$.

Proof. As in the proof of Proposition 4.9 we first show that for $f \in C_{c}^{\infty}(\mathbb{R} ; H)$ we have that

$$
\mathcal{S}_{\rho}(f)=\mathcal{S}_{\rho_{0}}(f)
$$


for each $\rho \geq \rho_{0}$. For doing so, define $u_{\rho_{0}}:=\mathcal{S}_{\rho_{0}}(f)$. Note that by Theorem 4.4 and since $\mathcal{S}_{\rho_{0}}(0)=0$, we derive that spt $u_{\rho_{0}}$ is bounded from below and hence, $u_{\rho_{0}} \in L_{2, \rho}(\mathbb{R} ; H)$. Thus, by Lemma 4.7 and Proposition 4.9 we derive that

$$
\begin{aligned}
u_{\rho_{0}}=\mathcal{S}_{\rho_{0}}(f) & \Leftrightarrow u_{\rho_{0}}=\mathcal{S}_{\rho_{0}, \text { aux }}\left(f+\left(\delta-\left(\mathcal{M}_{\rho_{0}}^{\prime}+\mathcal{N}_{\rho_{0}}\right) u_{\rho_{0}}\right)\right. \\
& \Leftrightarrow u_{\rho_{0}}=\mathcal{S}_{\rho, \text { aux }}\left(f+\left(\delta-\left(\mathcal{M}_{\rho}^{\prime}+\mathcal{N}_{\rho}\right) u_{\rho_{0}}\right)\right. \\
& \Leftrightarrow u_{\rho_{0}}=\mathcal{S}_{\rho}(f),
\end{aligned}
$$

which yields the claim. Since each function $f \in L_{2, \rho}(\mathbb{R} ; H) \cap L_{2, \mu}(\mathbb{R} ; H)$ can be approximated by one sequence of functions in $C_{c}^{\infty}(\mathbb{R} ; H)$ in both spaces $L_{2, \rho}(\mathbb{R} ; H)$ and $L_{2, \mu}(\mathbb{R} ; H)$, the assertion of the theorem follows.

\section{An Application to semistatic quasilinear Maxwell's equations}

We consider the following variant of Maxwell's equations on a domain $\Omega \subseteq \mathbb{R}^{3}$ in the semistatic case

$$
\begin{aligned}
\sigma E-\operatorname{curl} H & =-J, \\
\partial_{t, \rho} B+\operatorname{curl} E & =0,
\end{aligned}
$$

where $(E, H) \in L_{2, \rho}\left(\mathbb{R} ; L_{2}(\Omega)^{3}\right) \times L_{2, \rho}\left(\mathbb{R} ; L_{2}(\Omega)^{3}\right)$ denotes the electro-magnetic field and $B \in L_{2, \rho}\left(\mathbb{R} ; L_{2}(\Omega)^{3}\right)$ is the magnetic induction and $J \in L_{2, \rho}\left(\mathbb{R} ; L_{2}(\Omega)^{3}\right)$ is a given current density. The conductivity of the underlying medium is denoted by $\sigma$ and will be specified later. The equations are completed by the following constitutive relation linking $H$ and $B$

$$
(H(t), B(t)) \in \mathcal{Z} \quad(t \in \mathbb{R} \text { a.e. })
$$

for some $c$-maximal monotone bounded relation $\mathcal{Z} \subseteq L_{2}(\Omega)^{3} \times L_{2}(\Omega)^{3}$ with $c>0$ and the boundary conditions

$$
\begin{aligned}
n \cdot(J+\sigma E) & =0, \text { on } \partial \Omega \\
n \cdot B & =0, \text { on } \partial \Omega,
\end{aligned}
$$

where $n$ denotes the outward unit normal vector on $\partial \Omega$. It will turn out that in general the conditions (6) are not sufficient to ensure the uniqueness of a solution. We will come to this point later.

We start by introducing some variants of the operators curl and div.

Definition. We define the operator $\operatorname{div}_{0}$ as the closure of

$$
C_{c}^{\infty}(\Omega)^{3} \subseteq L_{2}(\Omega)^{3} \rightarrow L_{2}(\Omega):\left(\psi_{i}\right)_{i \in\{1,2,3\}} \mapsto \sum_{i=1}^{3} \partial_{i} \psi_{i}
$$


In the same way, we define the operator curl $_{0}$ as the closure of $C_{c}^{\infty}(\Omega)^{3} \subseteq L_{2}(\Omega)^{3} \rightarrow L_{2}(\Omega)^{3}:\left(\psi_{i}\right)_{i \in\{1,2,3\}} \mapsto\left(\partial_{2} \psi_{3}-\partial_{3} \psi_{2}, \partial_{3} \psi_{1}-\partial_{1} \psi_{3}, \partial_{1} \psi_{2}-\partial_{2} \psi_{1}\right)^{\top}$. Moreover, we define curl $:=\left(\operatorname{curl}_{0}\right)^{*}$.

Remark 5.1. In case of a smooth boundary $\partial \Omega$ the elements in $\Psi \in \operatorname{dom}\left(\operatorname{div}_{0}\right)$ can be characterised as those $L_{2}$-vector fields, whose divergence is an $L_{2}$-function and which satisfy the homogeneous Neumann boundary condition

$$
\Psi \cdot n=0 .
$$

Note that $\Psi \in \operatorname{dom}\left(\operatorname{div}_{0}\right)$ also makes sense even if $\Omega$ has a non-smooth boundary, where the classical homogeneous Neumann boundary condition could not be formulated via trace theorems. That is why we do not need to require any regularity on $\Omega$ and may use $\Psi \in \operatorname{dom}\left(\operatorname{div}_{0}\right)$ as a suitable generalised Neumann boundary condition.

In the same way, the elements in $E \in \operatorname{dom}\left(\operatorname{curl}_{0}\right)$ are those $L_{2}$-vector fields with a curl representable as an $L_{2}$-vector field and who satisfy the (generalised) electrical boundary condition

$$
E \times n=0 .
$$

Moreover, by definition

$$
\operatorname{dom}(\operatorname{curl})=\left\{E \in L_{2}(\Omega)^{3} ; \operatorname{curl} E \in L_{2}(\Omega)^{3}\right\},
$$

i.e., curl is the maximal realisation of the rotation as an operator on $L_{2}(\Omega)^{3}$.

Let $(E, B, H)$ be a solution of (44),(5) and (6) . From the first line of (4) we read of that $\sigma E+J$ has a vanishing $L_{2}$-divergence and since $\sigma E+J$ should satisfy ([6), we infer that indeed $E \in \operatorname{ker}\left(\operatorname{div}_{0}\right)$. In the same way, $B \in \operatorname{ker}\left(\operatorname{div}_{0}\right)$. We note that due to

$$
\operatorname{div}_{0} \operatorname{curl}_{0}=0
$$

it follows that $\overline{\operatorname{ran}\left(\operatorname{curl}_{0}\right)}$ is a closed subspace of ker $\left(\operatorname{div}_{0}\right)$. Hence, according to the projection theorem, we can decompose $\operatorname{ker}\left(\operatorname{div}_{0}\right)$ by (see also [11, 17])

$$
\begin{aligned}
\operatorname{ker}\left(\operatorname{div}_{0}\right) & =\overline{\operatorname{ran}\left(\operatorname{curl}_{0}\right)} \oplus\left(\operatorname{ran}\left(\operatorname{curl}_{0}\right)\right)^{\perp} \cap \operatorname{ker}\left(\operatorname{div}_{0}\right) \\
& =\overline{\operatorname{ran}\left(\operatorname{curl}_{0}\right)} \oplus \operatorname{ker}(\operatorname{curl}) \cap \operatorname{ker}\left(\operatorname{div}_{0}\right) .
\end{aligned}
$$

The set

$$
H_{N}:=\operatorname{ker}(\operatorname{curl}) \cap \operatorname{ker}\left(\operatorname{div}_{0}\right)
$$

is known as the set of harmonic Neumann fields. Note that $H_{N}$ is a closed subspace of $L_{2}(\Omega)^{3}$ and we denote the orthogonal projection onto $H_{N}$ by $P_{N}$. Additionally to the boundary conditions (6) we need to impose conditions on the values $P_{N}(\sigma E+J)$ and $P_{N} B$. For simplicity we set

$$
P_{N}(\sigma E+J)=P_{N} B=0 .
$$


For later reference, we summarise our so far found constraints:

$$
\begin{aligned}
\sigma E-\operatorname{curl} H & =-J, \\
\partial_{t, \rho} B+\operatorname{curl} E & =0, \\
(H(t), B(t)) & \in \mathcal{Z} \quad(t \in \mathbb{R} \text { a.e. }), \\
\sigma E+J, B & \in \operatorname{ker}\left(\operatorname{div}_{0}\right) \cap H_{N}^{\perp} .
\end{aligned}
$$

In order to incorporate the conditions (6) and (8), we need the following variant of the curl operator.

Proposition 5.2 ([17, Theorem 2.1]). Assume that $\operatorname{ran}\left(\operatorname{curl}_{0}\right)$ is closed. Then the operator

$$
\widetilde{\operatorname{curl}}: \operatorname{dom}(\widetilde{\operatorname{curl}}) \subseteq L_{2}(\Omega)^{3} \rightarrow L_{2}(\Omega)^{3}: W \mapsto \operatorname{curl} W
$$

with $\operatorname{dom}(\widetilde{\operatorname{curl}}):=\left\{W \in \operatorname{dom}(\operatorname{curl}) ; \operatorname{curl} W \in \operatorname{ran}\left(\operatorname{curl}_{0}\right)\right\}$ is selfadjoint.

The assumption that $\operatorname{ran}\left(\operatorname{curl}_{0}\right)$ is closed can for instance be ensured by assuming the compactness of the embedding

$$
\operatorname{dom}\left(\operatorname{curl}_{0}\right) \cap \operatorname{dom}(\operatorname{div}) \hookrightarrow L_{2}(\Omega)^{3} .
$$

We refer to [36, 16] for a proof of this result even on a class of Riemannian manifolds with non-smooth boundaries and to [37] for the case of a bounded domain whose boundary is allowed to have certain cusps.

We now come to the well-posedness result.

Theorem 5.3. Let $\sigma, \sigma^{\prime}, \kappa: C_{c}^{\infty}\left(\mathbb{R} ; L_{2}(\Omega)^{3}\right) \rightarrow \bigcap_{\mu \geq \rho_{0}} L_{2, \mu}\left(\mathbb{R} ; L_{2}(\Omega)^{3}\right)$ be evolutionary at $\rho_{0}>0$ such that

$$
(\sigma \varphi)^{\prime}=\sigma \varphi^{\prime}+\sigma^{\prime} \varphi \quad\left(\varphi \in C_{c}^{\infty}(\mathbb{R} ; H)\right)
$$

and there exists $c_{1}>0$ such that

$$
\operatorname{Re}\left\langle\left(\partial_{t, \rho} \sigma+\kappa\right) \varphi, \chi_{\mathbb{R}_{\leq a}} \varphi\right\rangle_{\rho} \geq c_{1}\left|\chi_{\mathbb{R}_{\leq a}} \varphi\right|_{\rho}^{2} \quad\left(\varphi \in C_{c}^{\infty}(\mathbb{R} ; H), a \in \mathbb{R}\right) .
$$

Moreover, assume that $\operatorname{ran}\left(\operatorname{curl}_{0}\right) \subseteq L_{2}(\Omega)^{3}$ is closed. Finally, let $\mathcal{Z} \subseteq L_{2}(\Omega)^{3} \times L_{2}(\Omega)^{3}$ be c-maximal monotone and bounded with $(0,0) \in \mathcal{Z}$. Then for each $\rho \geq \rho_{0}$

$$
\mathcal{S}_{\rho, \text { Max }}:=\left(\overline{\partial_{t, \rho}\left(\begin{array}{cc}
\sigma_{\rho} & 0 \\
0 & 0
\end{array}\right)+\left(\begin{array}{cc}
\kappa_{p} & 0 \\
0 & c
\end{array}\right)+\left(\begin{array}{cc}
0 & -\widetilde{\operatorname{curl}} \\
\widetilde{\operatorname{curl}} & \mathcal{Z}-c
\end{array}\right)}\right)^{-1}
$$

is a Lipschitz-continuous, causal mapping on $L_{2, \rho}\left(\mathbb{R} ; L_{2}(\Omega)^{3} \times L_{2}(\Omega)^{3}\right)$ and independent of $\rho$ in the sense of Theorem 4.10 .

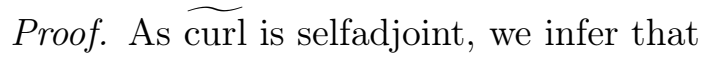

$$
\left(\begin{array}{cc}
0 & -\widetilde{\operatorname{curl}} \\
\text { curl } & 0
\end{array}\right)
$$


is skew-selfadjoint and hence, maximal monotone. Moreover $\mathcal{Z}-c$ is maximal monotone by Lemma 2.7 and bounded and thus,

$$
\mathcal{A}:=\left(\begin{array}{cc}
0 & -\widetilde{\operatorname{curl}} \\
\widetilde{\operatorname{curl}} & \mathcal{Z}-c
\end{array}\right)
$$

is maximal monotone by Corollary 2.15. Moreover, $(0,0) \in \mathcal{A}$ and hence, the operators $\mathcal{M}:=\left(\begin{array}{ll}\sigma & 0 \\ 0 & 0\end{array}\right), \mathcal{N}:=\left(\begin{array}{ll}\kappa & 0 \\ 0 & c\end{array}\right)$ and the relation $\mathcal{A}$ satisfy Hypotheses $\mathbb{C}$. Thus, the assertion follows by Theorem 3.1. Theorem 4.4 and Theorem 4.10

Remark 5.4. (a) A particular instance of operators $\sigma, \kappa$ satisfying the assumptions of the previous theorem are multiplication operator associated with a Lipschitz-continuous, bounded mapping $\sigma, \kappa: \mathbb{R} \rightarrow L\left(L_{2}(\Omega)^{3}\right)$ such that $\sigma(t)$ is injective, selfadjoint and uniformly strictly positive definite for each $t \in \mathbb{R}$. Those operators were considered in [24, 31].

(b) In case of $\mathcal{Z}$ being the inverse of a Lipschitz-continuous, $c$-monotone mapping, $\sigma$ a positive constant number and $\kappa=0$, the well-posedness result was already obtained in 12 employing a Galerkin approximation method.

To conclude this section, we show how the mapping $\mathcal{S}_{\rho, \text { Max }}$ indeed (at least formally 2 ) provides a solution of our problem (9).

Assume that $(E, B, H)$ satisfy (9). As $B$ and $\sigma E+J$ lie in $\operatorname{ker}\left(\operatorname{div}_{0}\right) \cap H_{N}^{\perp}$ by (6) and (8), we derive from (77) that

$$
B, \sigma E+J \in \operatorname{ran}\left(\operatorname{curl}_{0}\right)
$$

However,

$$
\partial_{t, \rho} B=-\operatorname{curl} E, \sigma E+J=\operatorname{curl} H
$$

and thus, $H, E \in \operatorname{dom}(\widetilde{\operatorname{curl}})$. Thus, we have the equations

$$
\begin{aligned}
\sigma E-\widetilde{\operatorname{curl}} H & =-J, \\
\partial_{t, \rho} B+\widetilde{\operatorname{curl}} E & =0 .
\end{aligned}
$$

Defining $\widetilde{E}:=\partial_{t, \rho}^{-1} E$ we get

$$
\begin{aligned}
\partial_{t, \rho} \sigma \widetilde{E}-\sigma^{\prime} \widetilde{E}-\widetilde{\operatorname{curl}} H & =-J, \\
B+\widetilde{\operatorname{curl}} \widetilde{E} & =0
\end{aligned}
$$

where we have used $\sigma \partial_{t, \rho} \widetilde{E}=\partial_{t, \rho} \sigma \widetilde{E}-\sigma^{\prime} \widetilde{E}$. Invoking (5), we derive that

$$
((\widetilde{E}, H),(-J, 0)) \in \partial_{t, \rho}\left(\begin{array}{cc}
\sigma_{\rho} & 0 \\
0 & 0
\end{array}\right)+\left(\begin{array}{cc}
-\sigma_{\rho}^{\prime} & 0 \\
0 & c
\end{array}\right)+\left(\begin{array}{cc}
0 & -\widetilde{\operatorname{curl}} \\
\text { curl } & \mathcal{Z}-c
\end{array}\right)_{\rho}
$$

\footnotetext{
${ }^{2}$ By assuming enough regularity of $J$, the computations could be made rigorous.
} 
On the other, following this argumentation backwards, we infer that (for $\kappa=-\sigma^{\prime}$ )

$$
(\widetilde{E}, H):=\mathcal{S}_{\rho, \operatorname{Max}}(-J, 0)
$$

yields a solution $(E, B, H)$ of (9) with $E:=\partial_{t, \rho} \widetilde{E}$ and $B=-\widetilde{\operatorname{curl}} \widetilde{E}$.

\section{References}

[1] P. Bénilan. Équations d'évolution dans un espace de Banach quelconque et applications. PhD thesis, Université Paris XI, 1972.

[2] H. Brezis. Operateurs maximaux monotones et semi-groupes de contractions dans les espaces de Hilbert. Universite Paris VI et CNRS, 1971.

[3] M. Crandall and A. Pazy. Nonlinear evolution equations in Banach spaces. Isr. J. Math., 11:57-94, 1972.

[4] K. J. Engel and R. Nagel. One-parameter semigroups for linear evolution equations. Graduate texts in mathematics. Springer, 2000.

[5] L. Evans. Nonlinear evolution equations in an arbitrary Banach space. Isr. J. Math., 26:1-42, 1977.

[6] S. Hu and N. S. Papageorgiou. Handbook of Multivalued Analysis, volume 1: Theory. Springer, 1997.

[7] A. Kalauch, R. Picard, S. Siegmund, S. Trostorff, and M. Waurick. A Hilbert space perspective on ordinary differential equations with memory term. J. Dyn. Differ. Equations, 26(2):369-399, 2014.

[8] T. Kato. Integration of the equation of evolution in a Banach space. J. Math. Soc. Japan, 5:208-234, 1953.

[9] K. Kobayasi, Y. Kobayashi, and S. Oharu. Nonlinear evolution operators in Banach spaces. Osaka J. Math., 21:281-310, 1984.

[10] D. F. McGhee and R. Picard. A note on anisotropic, inhomogeneous, poro-elastic media. Math. Methods Appl. Sci., 33(3):313-322, 2010.

[11] A. Milani and R. Picard. Decomposition theorems and their application to nonlinear electro- and magneto-static boundary value problems. Partial differential equations and calculus of variations, Lect. Notes Math. 1357, 317-340 (1988)., 1988.

[12] A. Milani and R. Picard. Weak solution theory for Maxwell's equations in the semistatic limit case. J. Math. Anal. Appl., 191(1):77-100, 1995.

[13] G. Minty. Monotone (nonlinear) operators in a hilbert space. Duke Math. J., 29, 1962. 
[14] G. Morosanu. Nonlinear evolution equations and applications. Springer, 2nd edition, 1988.

[15] R. Nagel. Extrapolation spaces for semigroups. RIMS Kokyuroku, 1009:181-191, 1997.

[16] R. Picard. An elementary proof for a compact imbedding result in generalized electromagnetic theory. Math. Z., 187:151-164, 1984.

[17] R. Picard. On a selfadjoint realization of curl and some of its applications. Ric. Mat., 47(1):153-180, 1998.

[18] R. Picard. A structural observation for linear material laws in classical mathematical physics. Math. Methods Appl. Sci., 32(14):1768-1803, 2009.

[19] R. Picard. A class of evolutionary problems with an application to acoustic waves with impedance type boundary conditions. In Spectral Theory, Mathematical System Theory, Evolution Equations, Differential and Difference Equations, volume 221 of Operator Theory: Advances and Applications, pages 533-548. Springer Basel, 2012.

[20] R. Picard and D. McGhee. Partial differential equations. A unified Hilbert space approach. de Gruyter Expositions in Mathematics 55. Berlin: de Gruyter. xviii, 2011.

[21] R. Picard, S. Trostorff, and M. Waurick. On evolutionary equations with material laws containing fractional integrals. Math. Methods Appl. Sci., 38(15):3141-3154, 2015.

[22] R. Picard, S. Trostorff, and M. Waurick. On some models for elastic solids with micro-structure. ZAMM, Z. Angew. Math. Mech., 95(7):664-689, 2015.

[23] R. Picard, S. Trostorff, and M. Waurick. Well-posedness via Monotonicity. An Overview. In W. Arendt, R. Chill, and Y. Tomilov, editors, Operator Semigroups Meet Complex Analysis, Harmonic Analysis and Mathematical Physics, number 250 in Operator Theory: Advances and Applications, pages 397-452. Springer International Publishing, 2015.

[24] R. Picard, S. Trostorff, M. Waurick, and M. Wehowski. On Non-autonomous Evolutionary Problems. J. Evol. Equ., 13(4):751-776, 2013.

[25] S. Trostorff. Well-posedness and causality for a class of evolutionary inclusions. PhD thesis, TU Dresden, 2011. URL: http://nbn-resolving.de/urn:nbn:de:bsz:14-qucosa-78325.

[26] S. Trostorff. An alternative approach to well-posedness of a class of differential inclusions in Hilbert spaces. Nonlinear Anal., Theory Methods Appl., Ser. A, Theory Methods, 75(15):5851-5865, 2012. 
[27] S. Trostorff. Autonomous Evolutionary Inclusions with Applications to Problems with Nonlinear Boundary Conditions. Int. J. Pure Appl. Math., 85(2):303-338, 2013.

[28] S. Trostorff. Exponential Stability for Linear Evolutionary Equations. Asymptotic Anal., 85:179-197, 2013.

[29] S. Trostorff. On Integro-Differential Inclusions with Operator-valued Kernels. Math. Methods Appl. Sci., 38(5):834-850, 2015.

[30] S. Trostorff. Exponential Stability and Initial Value Problems for Evolutionary Equations. Habilitation thesis, TU Dresden, 2018. URL: http://nbn-resolving.de/urn:nbn:de:bsz:14-qucosa-236494.

[31] S. Trostorff and M. Wehowski. Well-posedness of non-autonomous evolutionary inclusions. Nonlinear Anal., Theory Methods Appl., Ser. A, Theory Methods, 101:47$65,2014$.

[32] M. Waurick. G-convergence of linear differential equations. Z. Anal. Anwend., 33(4):385-415, 2014.

[33] M. Waurick. A note on causality in reflexive Banach spaces. Indag. Math., 26(2):404$412,2015$.

[34] M. Waurick. On non-autonomous integro-differential-algebraic evolutionary problems. Math. Methods Appl. Sci., 38(4):665-676, 2015.

[35] M. Waurick. On the continuous dependence on the coefficients of evolutionary equations. Habilitation thesis, TU Dresden, 2016. arXiv: 1606.07731.

[36] N. Weck. Maxwell's boundary value problem on Riemannian manifolds with nonsmooth boundaries. J. Math. Anal. Appl., 46:410-437, 1974.

[37] K. J. Witsch. A remark on a compactness result in electromagnetic theory. Math. Methods Appl. Sci., 16(2):123-129, 1993. 